\title{
1 Low-Energy Energetic Neutral Atom Imaging of 2 Io Plasma and Neutral Tori
}

$4 \quad$ Yoshifumi Futaana, Stas Barabash, Xiao-Dong Wang, Martin Wieser, and

5 Gabriella S. Wieser

6 Swedish Institute of Space Physics, Box 812, Kiruna 98128, Sweden.

7 Peter Wurz

8 University of Bern.

9 Norbert Krupp

10 Max Planck Institute for Solar System Research.

11 Pontus C:son Brandt

12 Applied Physics Laboratory, Johns Hopkins University.

\section{Corresponding author}

15 Yoshifumi Futaana, Swedish Institute of Space Physics, Box 812, Kiruna 98128,

16 Sweden.

17 E-mail: futaana@irf.se

18 Tel: $+46-980-79025$

19 Fax: +46-980-79050

\section{Abstract}

Io's plasma and neutral tori play significant roles in the Jovian magnetosphere. We present feasibility studies of measuring low-energy energetic neutral atoms (LENAs) generated from the lo tori. We calculate the

25 LENA flux between $10 \mathrm{eV}$ and $3 \mathrm{keV}$. The energy range includes the corotational plasma flow energy. The expected differential flux at Ganymede distance is typically $10^{3}-10^{5} \mathrm{~cm}^{-2} \mathrm{~s}^{-1} \mathrm{sr}^{-1} \mathrm{eV}^{-1}$ near the energy of the corotation. It is above the detection level of the planned LENA sensor that is to be flown to the Jupiter system with integration times of 0.01-1 seconds. The flux has strong asymmetry with respective to the lo phase. The observations will exhibit periodicities, which can be attributed to the Jovian magnetosphere rotation and

32 the rotation of lo around Jupiter. The energy spectra will exhibit dispersion

33 signatures, because of the non-negligible flight time of the LENAs from lo to the satellite. In 2030, the Jupiter exploration mission JUICE will conduct a LENA 
measurement with a LENA instrument, the Jovian Neutrals Analyzer (JNA). From the LENA observations collected by JNA, we will be able to derive characteristic quantities, such as the density, velocity, velocity distribution function, and composition of plasma-torus particles. We also discuss the possible physics to be explored by JNA in addition to the constraints for operating the sensor and analyzing the obtained dataset.

\section{Introduction}

\subsection{Io neutral and plasma tori}

lo, the innermost Galilean moon of Jupiter, introduces a large amount material of volcanic origin to the Jovian magnetosphere. As a result, the Jovian magnetosphere is composed of heavy ions (oxygen, sulfur, and their compounds). The strong and localized source of particles near lo creates characteristic spatial distributions of plasma and neutral particles in the Jovian magnetosphere, namely, the lo neutral and plasma tori (see, for example, reviews by Dessler 1983; Thomas et al. 2004; Schneider and Bagenal, 2007; and references therein).

The lo neutral torus (called also as neutral cloud) is a dense concentration of atoms and molecules of volcanic origin that has formed around the lo orbit. These atoms are gravitationally bound to Jupiter. The composition is mainly sulfur $(\mathrm{S})$ and oxygen $(\mathrm{O})$ atoms as well as their compounds (e.g. Thomas et al., 2004). Hydrogen $(H)$ atoms also exist, but the fraction thereof is small. Sodium $(\mathrm{Na})$ atoms are also present. $\mathrm{Na}$ is bright because of its $\mathrm{D}$-lines, but the fraction thereof is quite small; therefore the $\mathrm{Na}$ imaging is considered as a trace gas of more abundant compositions in the neutral torus (Mendillo et al., 2004). These atoms form a partial ring-like shape, which is associated with the short time scale of the loss of atoms via ionization (a few hours to 10s of hours depending on the processes; see e.g. Schneider and Bagenal., 2007) compared to the filling time of the full torus ( 150 hours assuming $2.5 \mathrm{~km} / \mathrm{s}$, the escape velocity of lo).

When the neutral components in the lo neutral torus are ionized, the charged particles are accelerated by the motional electric field in the 
66 corotational flow in the magnetosphere. The accelerated ions form a plasma 67 torus. The main components are (singly or multiply) charged $\mathrm{O}$ ions and $\mathrm{S}$ ions. $68 \mathrm{H}^{+}$ions are also present but the fraction thereof is less abundant. It is often 69 assumed 10\% [e.g. Thomas et al. 2004]. However, there are several 70 measurements showing much smaller fraction than 10\% (e.g. Wang et al., 71 1998a, b; Zarka et al., 2001). The plasma in the torus is corotating with the 72 Jovian magnetosphere. At the lo orbit, the corotational velocity of the plasma is $73 \sim 74 \mathrm{~km} / \mathrm{s}$. The revolution speed of lo is $\sim 17 \mathrm{~km} / \mathrm{s}$ in the same direction as the 74 corotational flow, so the relative plasma flow velocity is $\sim 57 \mathrm{~km} / \mathrm{s}$ (e.g. Thomas 75 et al., 2004). Even though the plasma source is more localized, because of the 76 longer residence time (Schneider and Bagenal, 2007) compared with the 77 rotation of the Jovian magnetosphere (9.925 hour) the plasma torus is nearly 78 axisymmetric in a plane, referred to as the centrifugal equator (Hill 1974), 79 defined by the rotation and magnetic dipole tilt. The tilt of the magnetic dipole 80 is $\sim 9.6^{\circ}$ (according to the so-called O4 model; Acuña and Ness, 1976), while 81 that of the centrifugal equator is represented as $2 / 3$ of the magnetic tilt (Hill 82 1974).

83 After the lo neutral torus was discovered via the D-line emission of $\mathrm{Na}$ 84 (Brown 1974) and lo plasma torus via S+ emission (Kupo et al., 1976), many 85 observations on lo tori have been performed. Historically, investigations of the 86 Io tori have been conducted via UV and IR spectroscopy (Herbert et al., 2001; 87 Mendillo et al, 2004; Nozawa et al., 2004; Steffl et al., 2004a; 2004b; 2006; 88 2008; Yoneda et al., 2010; 2014) and in situ plasma observations (Bagenal., 89 1994; 1997a; 1997b; Frank and Paterson, 2001). Attempts for understanding the 90 characteristics and the dynamics of logenic particles inside the Jovian 91 magnetosphere are not only driven by the interests in magnetospheric science, 92 but also the lo tori directly associate with the lo's volcanic activities (e.g. 93 Herbert et al., 2001; Mendillo et al., 2004, 2007; Nozawa et al., 2004; Yoneda 94 et al., 2010; 2014). The recent developed technique of energetic neutral atom 95 (ENA) imaging has the potential to provide information concerning the lo 96 plasma and neutral tori in an efficient manner.

\section{$97 \quad$ 1.2 Energetic neutral atom imaging in Jovian system}


ENA imaging is a technique for the remote investigation of the interaction

99 between space plasma and neutrals (e.g. Roelof and Williams, 1988;

100 Gruntmann, 1997). Several ENA instruments have been carried into space, and

101 the data collected by these instruments have been used to study the interactions

102 between space plasma and neutral gas (e.g. Burch, 2000, 2003; Krimigis et al.,

103 2002; Mauk et al., 2003; Brandt et al., 2005; Futaana et al., 2011; Goldstein

104 and McComas, 2013). More recently, ENA imaging has also been successfully

105 applied to investigations of the interactions between space plasma and the

106 Moon surface (e.g. Futaana et al., 2006; McComas et al., 2009; Wieser et al.,

107 2009; Schaufelberger et al., 2011; Futaana et al., 2012).

108 ENAs near Jupiter were detected by the Voyager 1 low-energy charged 109 particle (LECP) instrument. LECP is a sensor for high-energy plasma particles, 110 (Krimigis et al., 1977) but Kirsch et al. (1981) concluded the energetic neutral 111 atoms were a possible explanation of one of the observed signals. Cheng (1986) 112 calculated the interaction between plasma and neutral atoms in the inner 113 magnetosphere and concluded that the charge exchange makes a significant 114 contribution to the fluxes of energetic neutral particles. Based on the dedicated 115 ENA measurement performed by Cassini/INCA, Krimigis et al. (2002) reported 116 the firm evidence of high-energy ENAs (HENAs; >10 keV) emitted from the 117 Jupiter system. Later, Mauk et al. (2003) claimed that the observed ENAs in the 118 range of $50-80 \mathrm{keV}$ originated from a region slightly outside of the Europa orbit 119 (trans-Europa gas tori).

120 These previous observations of ENAs in the Jupiter environment were only 121 conducted in the high-energy regime (>10 keV). No low-energy ENA (LENA) 122 instrument (with a typical energy range of $10 \mathrm{eV}$ to a few $\mathrm{keV}$ ) has been 123 employed in the Jovian system, although there are some speculation for 124 studying moon-plasma interactions [Plainaki et al., 2010; Mililo et al., 2013; 125 Grasset et al., 2013]. This unexplored energy range of LENAs is expected to 126 provide us with valuable information regarding the characteristics of the lo tori, 127 their formation and loss mechanisms, and associated transport mechanisms of 128 iogenic materials. Whereas a fraction of the energy is carried by high-energy 129 particles in the Jovian system, the mass transfer, namely, the outward transport 
130 of iogenic materials, is dominated by low-energy particles (Bagenal and

131 Delamere, 2011). From this perspective, the low-energy particles are essential to

132 the characterization of the Jovian plasma environment.

$133 \quad 1.3$ Low-energy Energetic Neutral Atom imaging

134 Several LENA instruments have been used to investigate extraterrestrial 135 environments. For example, the Neutral Particle Imager and Neutral Particle 136 Detector were placed on board European Space Agency's (ESA's) Mars Express 137 to establish a basic understanding of the ENA environment of Mars (Barabash et 138 al., 2006). Replicas of these detectors were flown to Venus on the Venus 139 Express mission (Barabash et al., 2007). IBEX-Lo has imaged the heliopause 140 from the Earth orbit (McComas et al., 2009). The Chandrayaan-1 Energetic 141 Neutrals Analyzer (CENA) was placed into Moon orbit (e.g., Kazama et al., 142 2006; Barabash et al., 2009), providing evidence of interaction between the 143 solar wind and regolith surface. A replica of CENA, named Energetic Neutrals 144 Analyzer (ENA), will be flown to Mercury with the ESA-JAXA joint mission, 145 BepiColombo as a part of Mercury Plasma Particle Experiment (MPPE) on the 146 Mercury Magnetospheric Orbiter (MMO) spacecraft (Saito et al., 2010). 147 BepiColombo will also carry another LENA sensor, as part of the Search for 148 Exospheric Refilling and Emitted Neutral Abundances (SERENA) package, on the 149 Mercury Planetary Orbiter (MPO) spacecraft (Orsini et al., 2010). These aim to 150 image Mercury-plasma interaction by LENAs (e.g. Grande 1997; Barabash et al., 151 2001; Orsini et al., 2001; Massetti et al., 2003).

152 In 2022, ESA will launch its first Jupiter mission, JUpiter ICy moons 153 Explorer (JUICE; Grasset et al., 2013). JUICE is equipped with a complete 154 plasma package, Particle Environment Package (PEP), which includes a LENA 155 sensor named Jovian Neutrals Analyzer (JNA). The measurement principle of 156 JNA is identical to that of ENA and CENA (Kazama et al., 2006; Barabash et al., 157 2009).

158 In this paper, we estimate the LENA flux produced by charge-exchange 159 reactions between particles in the lo neutral and plasma tori to demonstrate the 160 feasibility of LENA observations from Ganymede orbit using existing LENA 
161 sensors. We assume simple models of the plasma and neutral tori. There are 162 several reasons for using the simple models; first, such models require only a 163 relatively short computational time for iterative calculation of the densities and 164 velocity distribution functions of plasma and neutral tori; second, the line-of165 sight integration for the LENA calculation will obscure details of small structures 166 in any case; and third, simple models provide morphologic pictures that are 167 useful for examining the application of this new technique of the LENA imaging 168 to unexplored environment. The model is static, although the lo plasma and 169 neutral tori are known to have temporal variations (e.g. Frank and Paterson et al., 170 2001; Herbert et al., 2001; 2003; Delamere et al., 2004; Nozawa et al., 2004; 171 Yoneda et al., 2009; Steffl et al., 2004a; 2006). This is because this paper aims 172 to understand the characteristics of LENA flux, particularly its intrinsic variations 173 of the LENA flux due to the measurement technique and its limitations. In 174 section 4, environmental variations, which are important for understanding 175 nature of the lo tori, will be discussed.

176 We calculate the LENA flux along the planned trajectory of the JUICE 177 spacecraft (Grasset et al., 2013) and discuss the observation capabilities of JNA 178 on board. In addition, we discuss the possible physics to be explored by JNA as 179 well as the constraints for operating the sensor and analyzing the obtained 180 dataset. We do not attempt to discuss the detailed physics of the lo tori in this 181 paper.

\section{Models}

The primary generation mechanism of LENAs near the lo tori is chargeexchange (also called "charge transfer"). Because of the co-existence of ions and neutral atoms, ions in the plasma torus $\left(\mathrm{X}^{\mathrm{n}+}\right)$, experience charge exchange with neutral atoms in the neutral torus $(\mathrm{Y})$ :

$$
X^{n+}+Y->X^{*}+Y^{n+}
$$

After the charge exchange, a neutral atom $\left(X^{*} ; E N A\right)$ and an ion $\left(Y^{n+}\right)$ are formed. The newly ionized particles begin to corotate. The newly born neutral

190 atoms have nearly the same energy (e.g. Rees, 1989) as that of the primary ion,

191 which corresponds to the corotational flow $(\sim 74 \mathrm{~km} / \mathrm{s}$ at the lo orbit, or $\sim 29$ 
$\mathrm{eV} / \mathrm{amu}$, namely $\sim 29 \mathrm{eV}$ for $\mathrm{H}, \sim 460 \mathrm{eV}$ for $\mathrm{O}$, and $\sim 920 \mathrm{eV}$ for S). The energies of these atoms are in the LENA energy range.

To calculate the expected LENA flux, one must construct models of the plasma and neutral tori. In addition, the cross sections for the relevant chargeexchange reactions are required. From those models, we can derive the expected ENA fluxes using the following formulation.

$$
f_{\mathrm{ENA}}\left(\overrightarrow{r_{0}}, \overrightarrow{v_{0}} ; t=0\right)=\int_{-\infty}^{0} f_{\text {plasma }}\left(\overrightarrow{r_{1}}, \overrightarrow{v_{1}} ; t_{1}\right) \cdot \sigma \cdot N_{n}\left(\overrightarrow{r_{1}}, t 1\right) d l
$$

Here, $f_{\mathrm{ENA}}$ is the ENA flux at the spacecraft position $\vec{r}_{0}$ and with the velocity $\vec{v}_{0}$ at a certain time $(t=0)$. The integral is taken from the spacecraft position back in time along the Kepler trajectory of the ENA. The distance along the trajectory is denoted by 1 . The plasma velocity distribution function $f_{\text {plasma }}$ is calculated for the position $\vec{r}_{1}$ and velocity $\vec{v}_{1}$ at generation, which are also determined from the Keplerian motion. $N_{n}$ is the neutral density, and $\sigma$ is the charge-exchange cross section. Details of the formulation are described in Appendix A.

Several sophisticated neutral-torus models have been proposed, and some of them reflect the physics very precisely (e.g. Smyth, 1992; Wilson et al., 2002; Smyth and Marconi, 2003). However, based on a simplified approach, we constructed our neutral-torus model in an analytic form. We modeled the density as a sum of two exponential distributions:

$$
n(R, \theta)=n_{0} \exp \left(-\frac{|\theta|}{\theta_{0}}-\frac{|R|}{R_{0}}\right)+n_{1} \exp \left(-\frac{|\theta|}{\theta_{1}}-\frac{|R|}{R_{1}}\right)
$$

$$
\theta_{i}= \begin{cases}\theta_{i h} & (\theta>0) \\ \theta_{i t} & (\theta<0)\end{cases}
$$

Here, $n(R, \theta)$ is the density at the distance $R$ from the lo torus and the separation angle $(\theta)$ from the Jupiter-lo line and $n_{0}$ and $n_{1}$ is the number densities for each distribution. The parameters $\theta_{0}, \theta_{1}, r_{0}$, and $r_{1}$ define the spread of the distribution. Different $\theta_{0}$ values are used to consider the asymmetry between the leading and trailing parts of the torus; namely, $\theta_{0 \mathrm{~h}}$ is used for the leading part $(\theta>0)$, and $\theta_{0 \mathrm{t}}$ is used for the trailing part $(\theta<0)$. The assumed values are as follows: $n_{0}=15000 \mathrm{~cm}^{-3}, R_{0}=0.072 \mathrm{Rj}, \theta_{0 \mathrm{~h}}=14.4^{\circ}, \theta_{0 \mathrm{t}}=3.6^{\circ}, n_{1}=50$ 
$\mathrm{cm}^{-3}, R_{1}=0.72 \mathrm{Rj}, \theta_{0 \mathrm{~h}}=86.6^{\circ}$, and $\theta_{0 \mathrm{t}}=14.4^{\circ}$. The typical density of the neutral

224 torus on a global scale is controlled by the second component (parameter $n_{1}$ ).

225 This value was selected to be very close to those used in previous studies, such

226 as Johnson and Strobel (1982) and Cheng (1986). Figure 1b compares the results

227 obtained using the simple model employed here and a numerical model

228 proposed by Smyth and Marconi (2003). Parameters in our simple model was

229 chosen to capture the general trend of the lo neutral torus. However, our model

230 underestimates the neutral density in most of the region. In some region, the

231 underestimation is by a factor of 10 or more. For our purpose of demonstrating

232 the feasibility of LENA imaging from Ganymede orbit, an underestimation of the

233 LENA flux is preferable rather than an overestimation. The neutral-torus model

234 does not include any composition $(\mathrm{H}, \mathrm{O}$ or $\mathrm{S})$ information. Although the neutral

235 composition only plays a key role to the amount of ENA via the cross section,

236 the composition information will be reflected into only the cross section model.

237 All neutral atoms are assumed to move at a speed that corresponds to Io's 238 orbital velocity.

239 For our plasma-torus model, the same argument for using a simple 240 analytical model is applied. In practice, the simplicity is of great benefit with 241 respect to the impact on computational resources because we need to calculate 242 the velocity distribution function of ions in the plasma torus (Appendix A). We 243 assumed the following model. The plasma-torus structure is independent of the 244 lo phase. Although there are a few models that better represent the Jovian 245 magnetosphere (e.g., Acuña and Ness, 1976; Dougherty et al., 1996; Connerney 246 et al., 1998), we employed a perfect dipole here. We used the dipole 247 component parameters from the $\mathrm{O} 4$ model, namely, the tilt of the dipole field is $2489.6^{\circ}$, pointing to the west longitude of $201.7^{\circ}$ in the System III coordinate system 249 (Acuña and Ness, 1976). However, it is known that the central plane (peak 250 density along a magnetic field line) of the plasma torus is located at the 251 centrifugal equator (Hill, 1974; Bagenal and Sullivan, 1981), which is between 252 the Jovian equator plane and magnetic equator plane. The maximum separation 253 between the centrifugal equator and magnetic equator is $\sim 3.2^{\circ}$ (Hill, 1974). 254 Therefore, we assumed the tilt of dipole axis to be $6.4^{\circ}$ to emulate the 
255 centrifugal equator instead of using the actual tilt of $\sim 9.6^{\circ}$ above. Indeed, the 256 change in the tilt angle of the dipole axis does not impact on the morphologic 257 views of the following results.

258 The total density of plasma-torus ions at the magnetic equator, $n_{\mathrm{L}}(\mathrm{r})$, was 259 taken from Divine and Garrett (1983) but slightly simplified inside the lo 260 distance (Figure 2b). Figure 2b also indicate values of other existing models: 261 Bagenal (1994), based on Voyager 1 data later validated by Galileo data; and 262 Bagenal and Delamere (2011) based on Galileo data. These models provide 263 slightly higher values especially in the inner region, but anyway, less than a 264 factor of 10 of the present model. Away from the equator, we modified the 265 density by the scale height of $\mathrm{H}$, $\exp (-\mathrm{z} / \mathrm{H})^{2}$, adopting Hill and Michel (1976) 266 and other authors (e.g. Bagenal et al., 1994; Bagenal and Delamere, 2011). 267 Here, $\mathrm{z}$ is the distance from the centrifugal equator along the field line and $\mathrm{H}$ $268(\mathrm{Rj})$ is $0.64 \times(\mathrm{Ti}(\mathrm{eV}) / \mathrm{Ai}(\mathrm{amu}))^{1 / 2}$. We assumed the bulk plasma to flow azimuthally 269 with the corotation velocity and the thermal speed, $v_{\mathrm{th}}$ corresponding to $70 \mathrm{eV}$ 270 for all species (Kivelson et al., 2004). For $\mathrm{O}^{+}$, this value corresponds to $v_{\text {th }} \sim 5.5$ $271 \mathrm{~km} / \mathrm{s}$. The assumed temperature of $70 \mathrm{eV}$ is slightly higher than the data 272 obtained from Voyager 1 PLS and Galileo PLS by a factor of 2-3 (see Figure 3 in 273 Beganel and Delamere, 2011), while the difference in thermal velocity is less 274 significant $(\sqrt{2}-\sqrt{3})$. We assume that the velocity distribution function is an 275 isotropic Maxwellian. This assumption significantly underestimates the high276 energy portion of the velocity distribution.

277 We also require cross-section models for the charge-exchange reactions.

278 There are three main neutral components $(\mathrm{S}, \mathrm{O}, \mathrm{H}$, and their compounds) and 279 their ions $\left(\mathrm{S}^{\mathrm{n}}, \mathrm{O}^{\mathrm{n}+}\right.$, and $\left.\mathrm{H}^{+}\right)$. Table 1 provides a list of the expected charge280 exchange cross sections (Johnson and Strobel, 1982; McGrath and Johnson, 281 1989) for a relative velocity of $\sim 60 \mathrm{~km} / \mathrm{s}$. The typical cross section for charge 282 exchange is $\sim(1-50) \times 10^{-16} \mathrm{~cm}^{2}$ in this energy range. Rather large deviations in 283 cross-section values have been reported in some cases. In such cases, we use 284 the lowest quantities, resulting in an underestimation of the LENA production. 285 We consider only the charge exchange reactions that produce LENAs directly. 286 For example, the reaction $\mathrm{O}^{++}+\mathrm{S} \rightarrow \mathrm{O}^{*}+\mathrm{S}^{++}$is considered between doubly 
charged ions (for example $\mathrm{O}^{++}$) and neutrals (e.g. S). There are of course the reaction of single electron transfer, namely, $\mathrm{O}^{++}+\mathrm{S} \rightarrow \mathrm{O}^{+}+\mathrm{S}^{+}$. However, since this reaction does not produce LENAs directly, we should not consider it.

To simplify the calculation, we calculated the total cross section as defined 291 below. First, we take the average of cross section for each parent ion species, 292 weighted by the fraction of neutral composition. This is the "convolved cross 293 section" in Table 1. This convolved cross section is the single value but 294 equivalent to the cross section of a parent ion for charge exchange process 295 through the multiple-species lo neutral torus. Here, the average relative 296 composition of S and O near the neutral torus is 1:4 (e.g., Johnson and Strobel, 297 1982). The $\mathrm{H}$ population is assumed to be $10 \%$ of the total assuming 298 stoichiometry. We neglect the molecular contributions to the cross-section 299 calculations, primarily because we could not find good reference data to 300 constrain the fraction and the charge exchange cross sections. Then, we again 301 take the weighted average to obtain the total cross section for each LENA 302 species, considering the parent ion's charge state. This is the cross section for 303 charge exchange production for specific LENA species from different charge304 state parent ions. The total calculated cross sections are 12,19 , and $9 \times 10^{-16}$ $305 \mathrm{~cm}^{2}$ for $\mathrm{H}, \mathrm{O}$, and $\mathrm{S}$, respectively (Table 1 ).

After calculating the LENA flux, we further evaluated if a conventional 307 LENA sensor can measure the lo torus image. As a reference instrument used in 308 this paper to calculate the LENA count rate to evaluate the measurement 309 feasibility, we consider the current design of the JNA instrument (hereafter 310 referred to as the JNA-prototype) prepared for the JUICE spacecraft. The JNA311 prototype has similar capabilities to the MPPE/ENA sensor and Chandrayaan312 1/CENA sensor (Kazama et al., 2006; Barabash et al., 2009). See Table 2 for a 313 summary of the specifications of the JNA-prototype.

\section{3. Model Results}

$315 \quad 3.1$ lo-torus LENA flux 
Figure 3 presents an image of simulated differential flux of the LENAs emitted from the lo tori. The oxygen and sulfur LENA images for the energy of $318210 \mathrm{eV}$ and $420 \mathrm{eV}$ (both for $\sim 50 \mathrm{~km} / \mathrm{s}$ ) is displayed as examples. The observer 319 is placed at the planned location of the JUICE spacecraft during its so-called 320 high-latitude imaging period (Grasset et al., 2013). During this period, the 321 spacecraft will have a slight inclination with respect to the equatorial plane. We 322 show here the case when the spacecraft was located at $23.3 \mathrm{Rj}$ at the latitude of $3236.5^{\circ}$ north from the Jovian equatorial plane.

324 The peak flux can reach $10^{5} / \mathrm{cm}^{2} \mathrm{~s} \mathrm{eV} \mathrm{sr}$ in this case. The shape of the 325 LENA image is similar to a slice of the lo plasma torus. The image exhibits a 326 very narrow angular distribution. One can see a shift in the peak signal toward 327 Jupiter by $\sim 10^{\circ}$. This shift is caused by the spacecraft velocity effect (aberration). 328 The simulated image clearly illustrates that the contribution of the spacecraft 329 motion cannot be neglected. The spacecraft is typically moving at $10 \mathrm{~km} / \mathrm{s}$, and 330 thus, the aberration effect is, at maximum, $\sim 10^{\circ}$ for $50 \mathrm{~km} / \mathrm{s}$ LENAs when the 331 ENA flow direction and the spacecraft velocity vector is perpendicular. When 332 they are parallel, this effect can be seen as a change in the observed ENA 333 velocity. The spacecraft velocity effect can be corrected by subtracting the 334 velocity of the spacecraft from the image in the actual data analysis (Appendix 335 A). Figure 3 also displays a strong signal from the "left" side of Jupiter in the 336 figure, where the north of Jupiter is drawn to be up. On this side, the lo tori 337 particles move toward the observer so that the generated ENAs fly to the 338 spacecraft. Although the corotation plasma undergoes charge exchange when 339 Io is located on the other side ("right" side in Figure 3) of Jupiter, the generated 340 LENAs will travel away from the spacecraft. Therefore, the instantaneous image 341 has a strong asymmetry. In the followings, we refer to the "left" side (strong 342 LENA flux) as toward side and the other as away side. The degree of toward343 away asymmetry depends on the temperature of the corotating plasma. More 344 asymmetry is expected for cooler plasma.

$345 \quad$ Figure 4 presents a series of LENA images taken along the planned orbit of 346 the JUICE spacecraft. Oxygen ENA images with the energy of $210 \mathrm{eV}$, same 347 condition as Figure 3, are shown as examples. The corresponding locations of 
the spacecraft are marked at the center of the figure. The LENAs from the lo tori are always seen from the toward side of Jupiter regardless of the relative position of the observer with respect to Jupiter and lo. The peak flux varies from $10^{3}$ to $10^{5} / \mathrm{cm}^{2} \mathrm{~s} \mathrm{sr} \mathrm{eV}$. In contrast, the intensity clearly depends on the relative position. We can detect a strong flux from the lo tori when the LENAs that are generated from the side with the corotating plasma move toward the spacecraft (equivalently, when lo is located on the toward side of Jupiter). The highest flux is obtained at Jupiter-lo-Observer angle of $\sim 165^{\circ}$ in this case (18 UT on May 20, 2031). This time is $\sim 8$ hours after the Jupiter-lo-Observer angle is $90^{\circ}$ (at 10 UT on May 20, 2031), when the maximum flux would be expected from the naïve prediction. This apparent discrepancy can be simply explained once we consider the flight time of the LENAs. Even if the maximum flux is produced when the Jupiter-lo-Observer angle is $90^{\circ}$, the LENAs take a few hours to reach to the spacecraft. For oxygen LENAs with a speed of $\sim 50 \mathrm{~km} / \mathrm{s}$, traversing the distance of over $15 \mathrm{Rj}\left(\sim 10^{6} \mathrm{~km}\right)$ from lo to the spacecraft takes $2 \times 10^{4} \mathrm{~s}(\sim 6 \mathrm{~h})$. As one might expect, the time lag depends on the velocity of the LENAs.

The time series of the LENA count rate to be observed by the JNA instrument (Table 2) at the JUICE location is shown in Figure 5. The spacecraft is assumed to point toward Jupiter, and the right edge of the aperture is aligned with Jupiter (gray dotted rectangle in Figure 3). The count rates of three species $(\mathrm{H}, \mathrm{O}$, and $\mathrm{S})$ are calculated by integrating the simulated LENA flux over the sensor pixel shown in Figure 3. The calculated peak count rate is 1-100 counts per second for all species. The $\mathrm{O}$ flux is most prominent because $\mathrm{O}$ is the dominant species in the plasma torus. The mass-dependent bulk energy of each species corresponds to the corotation velocity. Note that the peak energy appears to be higher than the corotational energy in Figure 4; this is mainly because the count rate is proportional, provided the g-factor is constant over the energy, to the energy flux, not the particle flux, so that the peak count rate shifts toward the higher energy.

The spectrogram displays several characteristic features. First, there is a clear $\sim 10 \mathrm{~h}$ periodicity. This periodicity arises from the rotation of the Jupiter's magnetosphere. The neutral torus moves up and down with respect to the 
plasma-torus structure, and the number of charge-exchange reactions varies accordingly, peaking when the magnetic equator is in line with the lo neutral torus. Second, there is a $\sim 50 \mathrm{~h}$ periodicity. This periodicity is associated with the rotation of lo (and the resulting lo neutral torus) around Jupiter with a period of $\sim 42.5 \mathrm{~h}$. Precisely speaking, this periodic feature is the synodic period of lo and the spacecraft. We can also observe the dispersion-like signatures because of the flight time of the LENAs from the torus to the spacecraft, which depends on their velocity. A lower LENA energy will cause the LENA to take longer to reach the spacecraft. The time scale of the dispersion is several hours.

\subsection{Loss of LENAs}

After being generated near the lo tori, some LENAs may be lost before arriving at the spacecraft. Thus, we must evaluate the importance of the loss processes. Here, three loss mechanisms are considered: electron impact ionization, charge exchange, and photoionization.

The probability of electron impact ionization $p_{\mathrm{ei}}$ is estimated as follows:

$$
p_{\mathrm{ei}}=\int_{0}^{\infty} n_{e}(l) \sigma_{\mathrm{ei}}(E) d l \sim\left\langle n_{e}\right\rangle \sigma_{\mathrm{ei}} L
$$

where $n_{\mathrm{e}}$ and $<n_{\mathrm{e}}>$ are the electron density and its average along the trajectory, respectively; I is the distance along the trajectory; $\sigma_{\mathrm{ei}}$ is the electron impact ionization cross section, which is a function of the impact energy; and $L$ is the typical scale length.

To determine the upper limit, let us consider the maximum cross section $\sigma_{\mathrm{ei}}=1.5 \times 10^{-16} \mathrm{~cm}^{2}$ (for $\sim 100 \mathrm{eV}$ electrons; Kim and Desclaux, 2002) and the maximum electron density $\sim 2000 \mathrm{~cm}^{-3}$ (near lo; Kivelson et al., 2004) over the traveling length of $L=15 \mathrm{Rj}\left(\sim 10^{11} \mathrm{~cm}\right)$. The resultant calculated probability of electron impact ionization is $p_{\mathrm{ei}}=<n_{\mathrm{e}}>\cdot \sigma_{\mathrm{ei}} \cdot L=3 \times 10^{-2}$. This value includes a large margin for error, and in reality, the loss probability must be considerably smaller.

407 The electron temperature is $\sim 10 \mathrm{eV}$ at lo's orbit (Kivelson et al., 2004), which is smaller than the oxygen ionization potential. In this case, the cross section for 409 ionization is negligible. The electron temperature reaches a few $100 \mathrm{eV}$ near 410 Europa or Ganymede, while in these regions, the electron density is one to two 
411 orders of magnitude smaller than the assumed density. The high-energy tail 412 (including radiation-belt energy) of the electrons contributes to the ionization, 413 but the cross section is again much smaller (e.g. Itikawa and Ichimura, 1990).

414 The generated LENAs can also experience charge exchange once again 415 with other ions, thereby becoming re-ionized. The generated ions begin 416 corotating around Jupiter, and the generated neutral atoms should be 417 considered as ENAs, but their velocities become consistent with the local 418 corotational speed. A similar calculation can be performed as for electron 419 impact ionization, and the probability of charge exchange is

$$
p_{\mathrm{cx}}=\int_{0}^{\infty} n(l) \sigma_{\mathrm{cx}} d l \sim\langle n\rangle \sigma_{\mathrm{cx}} L
$$

The density, $<n>$, is the density of the plasma and takes a maximum value of $2000 \mathrm{~cm}^{-3} ; \sigma_{c x}$ can reach as high as $10^{-15} \mathrm{~cm}^{2}$. If the distance $L$ is taken along 424 the whole travelling length of $15 \mathrm{Rj}\left(\sim 10^{11} \mathrm{~cm}\right)$, the loss fraction calculated using these values is 0.2. Again, the obtained value is an upper limit with a 426 large margin for error, particularly because of the plasma and neutral densities 427 quickly decreases from the lo orbit. Plasma density drops by one order beyond $4282 \mathrm{Rj}$ (Figure 2b). The neutral density drops much quicker (Figure 1). Therefore, 429 the loss fraction should be much lower in reality, more than by one order $430 \quad(<<0.02)$.

431 Photoionization is another potential loss process. The ionization potentials 432 for the relevant species are $\sim 10 \mathrm{~V}(10.4 \mathrm{~V}$ for $\mathrm{S}$ and $13.6 \mathrm{~V}$ for $\mathrm{O}$ and $\mathrm{H})$, and 433 thus, the impinging photons should have energies of more than $10 \mathrm{eV}$ (wave 434 length $<100 \mathrm{~nm} ; \mathrm{V}-\mathrm{UV}, \mathrm{E}-\mathrm{UV}$ and shorter). The only possible source of such 435 short-wavelength photons is the Sun. At the typical solar irradiance, the power 436 in this wavelength range is rather constant at $10^{-4} \mathrm{~W} \mathrm{~m}^{-2} \mathrm{~nm}^{-1}$ at $1 \mathrm{AU}$ (e.g., 437 Schmidtke et al., 2006). Thus, the total photon flux can then be estimated to be $438 \sim 3 \times 10^{8} \mathrm{~cm}^{-2} \mathrm{~s}^{-1}$, and at the Jupiter orbit $(\sim 5.9 \mathrm{AU})$, it is reduced to $\sim 10^{7} \mathrm{~cm}^{-2} \mathrm{~s}^{-1}$ 439 integrated over wavelengths of $<100 \mathrm{~nm}$. The cross section of the 440 photoionization reaches a maximum of $\sim 2 \times 10^{-17} \mathrm{~cm}^{2}$ at $65-70 \mathrm{~nm}$ for oxygen 441 atoms, according to the EUVAC model (Richards et al., 1994). To obtain an 442 upper limit, we simply take the peak value over the entire wavelength range, 
443 which yields a loss rate of $\sim 2 \times 10^{-10} \mathrm{~s}^{-1}$, and thus, the minimum LENA lifetime is 444 estimated to be $5 \times 10^{9} \mathrm{~s}$. This lifetime is sufficiently long compared with the 445 flight time of LENAs $\left(10^{4}-10^{5} \mathrm{~s}\right)$ that we can neglect the loss caused by 446 photoionization.

447 In conclusion, ENA loss between the lo tori and the observer is not very 448 large, and thus is not critical for lo-tori LENA imaging. Indeed, because the 449 energy range of interest in this work is rather restricted, the energy dependence 450 of all loss mechanisms is weak so the shape of the energy spectrum is conserved. 451 Thus, losses of LENAs do not significantly impact the feasibility of LENA 452 imaging and associated scientific achievements.

\subsection{Sensor capabilities}

The expected differential flux is typically $\sim 10^{3}-10^{5} \mathrm{~cm}^{-2} \mathrm{~s}^{-1} \mathrm{sr}^{-1} \mathrm{eV}^{-1}$

455 (Figures 3 and 4) at the Ganymede distance. This flux corresponds to $\sim 1-100$ counts per second by considering the JNA-prototype performance at the JUICE planned orbit (Figure 5). However, one difficulty of collecting instantaneous observations of Io torus ENA from the Ganymede distance is that lo LENAs have a narrow angular beam-like structure with a full width half maximum of $<5^{\circ}$, which is rather small in comparison with the JNA-prototype angular resolution of $\sim 30 \times 5^{\circ}$. Thus, fine structures may be hidden in one angular pixel. Better angular resolution is required to resolve these fine structures. Although the peak

463 count rate is enough in the detectable level, a longer integration time may be 464 beneficial for investigating the energy spectrum or the spatial distributions. For 465 example, a change in the global morphology of LENA specta, such as the 466 dispersion relations seen in Figure 5, can be identified even when integrating 467 over several to tens of minutes. Let us suppose here a 10-min integration (during 468 which the spatial configuration would not change significantly) with 8 energy 469 steps, then $\sim 75 \mathrm{~s}$ integration is possible for each energy step, and the 470 corresponding number of counts will be increased to 75-7500 counts. Indeed, a

471 longer time integration will also aid in decreasing the telemetry and in reducing 472 the ambiguity of background counts that originate from the intense Jovian 473 radiation environment. 


\section{Scientific objectives}

In this section, we discuss what information can be obtained from LENA measurements of the lo tori and what scientific objectives we can address using these data. Table 3 summarizes the quantities that LENA data can (and cannot) provide. Detailed explanations and justifications are discussed in the following.

\section{1 lo-tori characterization}

LENA data can provide physical quantities that describe the plasma torus. The shape of the energy spectrum of the LENAs and their chemical (mass) composition can be obtained. The energy spectra directly reflect those of the lo torus plasma, thereby, providing information that is critical to understanding the characteristics and dynamics of the plasma in the plasma torus. The velocity of the plasma in the torus and its temperature can also be calculated using the LENA measurement. The absolute densities of the neutral and plasma tori cannot be easily deconvolved from the LENA dataset alone because the LENA data provide only a line-of-sight integral of a convolution of both densities.

The mass information reflects the chemical composition in the plasma torus. The mass composition is strongly influenced by generation and loss mechanisms (e.g. Frank and Paterson, 2001; Delamere et al., 2004; Hansen et al., 2005; Steffl et al., 2006). For example, if the $\mathrm{O}^{+} / \mathrm{S}^{+}$ratio is two, one may infer, under the stoichiometric assumption, that the lo plasma torus originates from volcanic lo materials $\left(\mathrm{SO}_{2}\right)$ and that the loss mechanism does not depend on species. Deriving the mass fraction of $\mathrm{H}^{+}$will aid in understanding the dynamics of protons in the Jovian magnetosphere. Proton distribution in the lo torus is not a well constrained information (e.g. Thomas et al. 2004). Although the proton fraction has been assumed $10 \%$, there are several measurements showing much smaller fraction than 10\% (e.g. Wang et al., 1998a, b; Zarka et al., 2001). The discrepancies indicate strong spatial or temporal variations. LENA imaging can reveal proton fraction and its spatial distribution instantaneously. 
The charge state of ions in the plasma torus, e.g., $\mathrm{S}^{+}$and $\mathrm{S}^{++}$, might be 504 derived from the different shapes of the energy spectra. Because of the $\mathrm{M} / \mathrm{q}$ 505 difference between different charge states, different charge-state ions of the 506 same species can have different energy spectra (e.g., temperatures). For example, $507 \mathrm{~S}^{++}(M / q=16)$ is expected to have a temperature similar to $\mathrm{O}^{+}(M / q=16)$ but 508 different from $\mathrm{S}^{+}(M / q=32)$, if one attributes the ion temperatures in the plasma 509 torus to the pickup process. It is also believed that the lo plasma torus is not in 510 chemical equilibrium (e.g., Thomas et al., 2004), implying that there is no 511 reason that $\mathrm{S}^{+}$and $\mathrm{S}^{++}$should have the same spectral shape. The separation of 512 charge states using the LENA velocity distribution functions may be a realistic 513 goal.

514 Whereas the LENA energy spectra and LENA mass spectra reflect the 515 plasma-torus characteristics well, the neutral-torus characteristics are more 516 difficult to obtain. In theory, the velocity of the neutrals in the torus and the 517 mass composition are convoluted in the cross section. However, to extract this 518 information from the actual data is quite difficult. There is an uncertainty in the 519 velocity dependence of the cross section. The charge-exchange cross sections 520 associated with $\mathrm{S}$ are particularly difficult to obtain in laboratory measurements, 521 as $\mathrm{S}$ tends to react chemically with ambient gas and instrumentation. Thus, we 522 must refer to theoretical calculations (e.g. Johnson and Strobel, 1982). Other 523 complimented observations (Section 5.2) or modelling effort to restrict the LENA 524 data interpretation (Section 5.2) would be more realistic way of investigation.

\section{$525 \quad$ 4.2 Short-term variations}

Short-term temporal variations (or, in particular, abrupt variations) in the 527 plasma and neutral tori, if they occur, can result in corresponding variations in 528 the LENA flux. From continuous time series of LENA energy and mass spectra, 529 one can identify which of the tori is responsible for the LENA variation. This 530 separation can be achieved because of the different responses of the LENA flux 531 to the changes in the plasma and neutral tori. When the density of the neutral 532 torus increases abruptly for some reason, the LENA flux increases regardless of 533 energy and mass; more precisely speaking, the change would be proportional 
534 the cross sections to the relative, but it can be distinguished. In contrast, there is

535 a high likelihood that a temporal variation in the plasma torus would induce 536 modifications only at specific energies and/or masses. For example, in the case 537 of sporadic injection of plasma into the plasma torus, the LENA image would be 538 affected only at the corresponding energy and/or mass of the injection.

539 Another possible phenomenon for investigation is the hypothetical $\mathrm{O}$ and $540 \mathrm{~S}$ streams. $\mathrm{Na}$ is known to exhibit a short temporal scale $(<10$ hours $)$ and 541 relatively small spatial scale (a few lo radii) stream (e.g., Schneider et al., 1991; 542 Wilson et al., 2002; Thomas et al., 2004; Schneider and Bagenal 2007). 543 Although the existence of similar streams for $\mathrm{O}$ and $\mathrm{S}$ is thought to be less 544 plausible because of the different characteristics (timescales and chemical 545 reactions) of $\mathrm{Na}, \mathrm{O}$, and $\mathrm{S}$, LENA imaging may provide information to place 546 limit on the existence of fast $\mathrm{O}$ and $\mathrm{S}$ stream in the near lo environment.

\section{$547 \quad 4.3$ Long-term variations}

From Galileo observations, the plasma-torus density is known to vary 549 significantly over time. Frank and Paterson (2001) claimed that the density of 550 the lo plasma torus during the 1995 flyby was three to four times higher than 551 during the other flybys in 1999 and 2000. One of the hypothetical sources of 552 enhancement of the lo tori is variable volcanic activity. Indeed, the UV 553 measurement performed by Cassini indicates a long-term (week to month) 554 change in the tori, which may be attributed to volcanic activity (Schneider and 555 Bagenal, 2007). Ground-based observations indicate systematic long-term 556 correlations between the volcanic activities and the emission of IR (Nozawa et 557 al., 2004; Yoneda et al., 2010).

558 If volcanic activities increase, the neutral-torus density initially increases. 559 After a period of time (the ionization time scale depends on the mechanisms, 560 being typically a few hours; e.g., Smyth and Combi, 1988; Schneider and 561 Bagenal., 2007), the higher-density neutrals become ionized to form a denser 562 plasma torus. The higher density plasma torus may retain more than 10 s of days 563 (e.g. Schneider and Bagenal, 2007). We can monitor the evolution of the lo tori 564 by tracking these temporal changes; first ENA flux increases almost independent 
565 of the species and energies because of the increase of neutral component, 566 followed by species-by-species energy dependent increase due to the increase 567 of the plasma composition, depending on the generation and loss mechanisms.

\section{$568 \quad 4.4$ Transport}

ENAs are considered to be one of the transport mechanisms of materials from the lo orbit outward. Because of Io's volcanic activity $\sim 10^{28}$ particles are introduced into the neutral torus per second, and (1-2) $\times 10^{26}$ ions are transported out of the Jovian system per second via the charge-exchanged ENAs in the highenergy domain of the Cassini/INCA sensor (Krimigis et al., 2002). These authors also inferred that the total escape flux, including lower energies, is $10^{27} / \mathrm{s}$, which is $10 \%$ of the generated flux. The direct measurement of the LENAs will provide a more precise estimate of the loss rate from the torus via the charge exchange.

Figure 6 shows the LENA distribution seen on the global scale of the Jovian magnetosphere. Each dot corresponds to a LENA, which are emitted from lo tori with the velocity of the corotation and a thermal spread of $5.5 \mathrm{~km} / \mathrm{s}$ (T 70 eV for $\mathrm{O}$ is assumed). The global LENA distribution forms a spiral shape, although each LENA is traveling almost radially. This spiral shape is conceptually similar to the Parker spiral of the solar wind magnetic field or water ejected from a rotating sprinkler. To produce this plot, we did not consider the Jovian magnetic field tilt for simplicity. Inclusion of the tilt will embed a $\sim 10$ hour modulation (corresponding to $\sim 85^{\circ}$ in the azimuthal direction).

The transfer from lo is not axisymmetric inside the Jovian magnetosphere. If one is located at a certain point, the LENA flux from the lo torus is measured as an intermittent flow. In contrast, the thermal spread of the lo plasma and distribution of the generation point (although it is close to lo position) will dissipate the shape of the spatial distribution in the very far tail (> 150 Rj with the parameters used in this calculation). This image can be complemented by the "neutral nebula" concept formulated by Krimigis et al. (2002), in which they suggested a disk-like confinement of LENAs in the Jovian equatorial plane generated by lo's (or other moons') tori. However, the view provided in Figure 6 indicates a spiral structure inside the nebula. 


\subsection{Lag of the plasma corotation}

Based on Voyager 1 plasma sensor data, McNutt et al. (1979) reported that the Jovian magnetosphere exhibits a slightly slower than "perfectly" rigid corotational flow. This velocity lag has been identified even at lo's distance. The in situ plasma measurements collected by Galileo indicated that the lag is $\sim 2-$ $10 \mathrm{~km} / \mathrm{s}$, with an average lag of 2-3 km/s (Frank and Paterson, 2001). Higher velocities than the corotational velocity have never been observed. Hill (1980) suggested that the lag is the effect of mass loading. Another suggested mechanism is the hypothetical latitudinal differential rotation of the Jovian magnetosphere (Dessler 1985). Long-term observations permitting the characterization of the velocity of the plasma torus can restrict the possible slow-down mechanisms of the plasma torus in the vicinity of lo. If we can point the FOV of JNA perpendicular to the magnetic equatorial plane (perpendicular to the pixel shown in Figure 3), the collected LENA data along the FoV will provide the latitudinal difference in the torus velocity. This operation will test the differential-rotation hypothesis. By investigating the correlations between the torus densities and the velocity lag determined from LENA measurements, one may also constrain the momentum transfers between the plasma and neutral tori, which directly contribute to the mass loading.

The typical velocity lag is $2-3 \mathrm{~km} / \mathrm{s}$, which corresponds to $\sim 3-4 \%$ of the corotational velocity (and to $\sim 5-8 \%$ of the energy). Distinguishing an energy difference of $<10 \%$ from JNA data may not be a straightforward analysis, but it should still be possible if one employs proper analysis methods. For example, as in Futaana et al. (2013), it is possible to clearly distinguish the LENA flux for parametric temperatures of $\sim 85 \mathrm{eV}$ from that for $75 \mathrm{eV}$ (a $\sim 10 \%$ difference in energy). When the velocity lag is as large as $5-10 \mathrm{~km} / \mathrm{s}(\sim 7-13 \%$ of the corotational flow), the energy difference becomes $\sim 15-25 \%$, and the possibility of the discrimination becomes more promising.

\section{Operation, Collaboration, and Data analysis}

\subsection{Challenges}


Although a sufficient count rate of LENAs from the lo tori is expected, operating the LENA instrument during the real mission and analyzing the 628 obtained data pose a considerable challenge. The limited field of view (FOV) is the most problematic issue. The FOV of the existing LENA sensors is a singlepixel camera (McComas et al., 2009) or a fan-shaped 1-D aperture (Barabash et al., 2009), and thus, the angular acceptance is very small. When this small acceptance is combined with the relatively small angular spread of the lo-torus LENA image, a small shift in the aperture results in the loss of the LENA signal peak. Although we may know the predicted attitude of the spacecraft beforehand in the practical operation, pointing toward the lo torus is not a simple task. The spacecraft velocity (ram velocity) is not negligible for lo-torus LENA imaging (Figures 3 and 4), and the optimal compensating angle depends on the energy of LENAs. Thus, there is no unique optimal solution for spacecraft pointing that captures the maximum fluxes for all energies. A rather good argument here is that the data analysis after data reception is simpler because all information necessary to correct the aberration effect is known.

The second problem is the LENA flight time. The typical measurement of the lo torus by the JUICE spacecraft will be performed at the Ganymede orbit $(\sim 15 \mathrm{Rj})$ or farther. For the LENA with a velocity of $74 \mathrm{~km} / \mathrm{s}$, the flight time is $\sim 1.4 \times 10^{4} \mathrm{~s}$. Because lo rotates around Jupiter in $\sim 42.5 \mathrm{~h}$, lo moves more than $30^{\circ}$ in the time it takes for the LENAs to reach the instrument. For the instrumental operation, we should refer not to the Io's position at the operating time but the position several hours before depending on the LENA energy. As shown in Figure 4, we can clearly see a peak emission a few hours after the apparent optimal condition.

Third, the gravitational force exerted by Jupiter affects the LENA trajectories and causes them to become to ballistic trajectories. The degree of bending depends on the velocity, but the low-velocity LENAs are most strongly affected. The gravitation force is a well-known parameter, and its influence is smaller than that of the flight time discussed above; however, the gravity force must be properly considered for precise analysis of the obtained data. 
Fourth, the charge-exchange cross sections are still not well constrained. They also depend on the relative velocities between the plasma and neutral particles in the tori. However, the dependency is not very strong within the LENA energy range. For example, for the $\mathrm{O}^{+}-\mathrm{O}$ interaction, the variation with energy is approximately a factor of two to three between $40 \mathrm{eV}$ and $10 \mathrm{keV}$ (Lindsay and Stebbings, 2005). Nevertheless, when one calculates the physical quantities of the tori (e.g., the density, composition, and other quantities) from the obtained data, the uncertainty of the cross sections directly impacts the uncertainties of those quantities.

\subsection{Synergy with Other Measurements}

HENAs are also expected from high-energy particles in the plasma torus. JNA will be accompanied by the Jovian Energetic Neutrals and Ions (JENI) sensor, which is also included as a part of the PEP suite to detect HENAs. Their energy range is planned to cover $0.5-300 \mathrm{keV}$. HENAs will be detected even when fewer LENAs are detected (when lo is on the away side) because of the high thermal velocity of the original ions. HENAs provide information regarding characteristics of the lo torus plasma and Io's radiation environment, and moreover, they may preserve information concerning acceleration mechanisms. Thus, the combination of LENA and HENA time-series data will permit the investigation of the evolution of the acceleration of plasma in the plasma torus. In addition, JENI is capable of 2-D imaging. The simultaneous JENI imaging will extend the observable velocity and mass space of ENAs.

Another related science target to be investigated via the synergy between LENA and HENA data is the trans-Europa gas tori (Mauk et al., 2003). Based on a HENA (50-80 keV) image obtained using the Cassini/INCA instrument, the existence of Europa gas tori (composed of $\mathrm{H}$ and $\mathrm{O}$ ) has been suggested, although this instrument has found no evidence of distinct lo-tori related HENAs. The corotational velocity at the Europa orbit is $\sim 150 \mathrm{~km} / \mathrm{s}$, and thus, the core component $(\mathrm{H}$ and $\mathrm{O})$ of ENAs generated in the Europa-tori also falls into the LENA energy range. Sulfur may reach $\sim 4 \mathrm{keV}$, which is almost the upper limit of the JNA sensor. A LENA measurement will provide complimentary evidence of 

the existence of trans-Europa gas tori. Distinguishing between the lo and Europa tori from LENA data alone will not be simple because the JNA-prototype has a rather coarse angular resolution (Table 2). However, it will still be possible to distinguish between the lo and Europa tori using the energy spectra and their periodic features, the dispersion signatures and the mass spectra. The energy spectrum of LENAs from lo should have a peak at $\sim 74 \mathrm{~km} / \mathrm{s}$, whereas the LENAs from Europa should have a peak at $\sim 150 \mathrm{~km} / \mathrm{s}$, corresponding to their respective corotation velocities. In addition, the periodicity of the ENA signals, as seen in Figure 5, must differ according to the moons' rotation period.

LENA imaging can be compared with observations of UV emission to gain further understanding of the lo tori. The UV and LENA measurements are both imaging, so that the obtained data can be used complimentarily. A difference of characteristics is the LENA line-of-sight (trjaectory) is twisted by the gravity of Jupiter; therefore the different slice of of the information may be obtained. If the neutral (plasma) density is derived via the UV spectroscopy, the plasma (neutral) density can be derived using a LENA sensor, in principle. Another difference is the emission signature. Since the photon is emitted isotropically, the obtained image will be fundamentally different from the highly directional emission of LENAs. For example, the lo-torus UV emission measured using the EUVE satellite exhibits a dawn-dusk asymmetry, in which the dusk side is stronger, and there is no apparent lo-phase dependence (Gladstone and Hall, 1998). In contrast, the Voyager UV measurement did indicate an lo-phase dependence (Sandel and Broadfoot, 1982). These features in the UV images differ from the predicted LENA images, which exhibit a clear toward-away asymmetry in which the toward side is stronger. Although the toward-dusk asymmetry of the lo tori is essential to understanding the generation and loss mechanisms and the dynamics of particles in the tori, placing constraints on the asymmetry using the LENA dataset is a difficult task because the peak flux of the LENAs on the away side will never reach the spacecraft (because of the strongly directional flow of LENAs). This indicates that the LENA imaging have a information gap depending on the lo position: Recurrence of the measurement is $\sim 50$ hour for LENAs, so that any changes below the time scale in LENA signatures are hardly attributed 
720 to either the change in the neutral or the plasma tori. UV imaging will help to 721 fill the gap of this information. Indeed, variations in such time scales have been 722 reported. Many authors (e.g. Sandel and Dessler, 1988; Brown 1995; Frank and

723 Petersen, 2001; Nozawa et al., 2004; Steffl et al., 2006) argued that a typical 724 variation in tori characteristic period could be modulated by the so-called 725 System IV period ( 10.2 hours). Cassin UV spectroscopy reported the 726 enhancement of the torus emission power by $20 \%$ for about 20 hours (Steffl et 727 al., 2004). Such phenomena in these time scales are difficult to investigate only 728 from LENA imaging.

729 Synergy with state-of-the-art data-analysis algorithms should also be 730 mentioned here. The JNA-prototype provides angular separation in only one 731 direction of the angle, which will limit the intuitive understanding of the LENA 732 signal. We may require the aid of numerical models and/or inversion techniques. 733 Such inversion techniques have been applied beginning with the early ENA 734 observations (e.g., Demajistre et al., 2004; Galli et al., 2008a; 2008d; Nakano et 735 al., 2008). Similar techniques should be optimized for each scientific objective, 736 implemented and validated for interpreting LENAs that originate from the lo tori, 737 considering all the affecting parameters (spacecraft velocity, flight time, and 738 gravity).

\section{$739 \quad 5.3$ Impact on spacecraft and mission design}

740 One benefit of LENAs compared with in situ measurements is that LENAs 741 can enable the remote sensing of particles in the lo tori. Without actually 742 entering to Io's orbit, we can investigate the near-lo neutral and plasma

743 environment. A harsher will make the spacecraft and instrument designs more 744 challenging. In addition, the background caused by radiation cannot be avoided, 745 so one requires multiple-coincidence detection systems or longer time integrals 746 to improve the signal-to-noise ratio for an in situ plasma sensor. These 747 approaches typically lower the instrument sensitivity by a factor of 10-100.

\section{6. Summary}


We have calculated the flux and expected count rate of energetic neutral atoms in the low-energy domain (LENA) from the lo plasma and neutral tori. 751 The expected total flux is $10^{3}-10^{5} \mathrm{~cm}^{-2} \mathrm{~s}^{-1} \mathrm{sr}^{-1} \mathrm{eV}^{-1}$, if one measures from the 752 Ganymede orbit. The flux is comparable to or above the one-count-per-second 753 level of the LENA sensor that has been designed for Jupiter exploration.

754 The time scale of the variation in the global morphology of the LENA flux 755 is the rotation time of the Jupiter magnetosphere, which is $\sim 10 \mathrm{~h}$. A longer time 756 scale of $\sim 50 \mathrm{~h}$, which corresponds to the synodic period of lo and the spacecraft, 757 is also expected. Because of the flight time of LENAs, the LENA energy spectrum 758 exhibits dispersion signatures, typically with a time scale of several hours. The 759 angular spread is rather confined, and thus, the LENA sensor should be improved to achieve higher angular resolution for resolving fine structures.

In 2030, the Jupiter exploration mission JUICE will conduct the first Jovian

762

763

764

765

766 LENA measurement using a LENA instrument, JNA. From LENA observations, we can derive the characteristic quantities such as the energy spectra, density, velocity, and the composition of plasma-torus particles. We can further investigate the temporal variations (both short- and long-term) of the tori, the transport of materials, and acceleration mechanisms.

\section{Acknowledgments}

The authors appreciate the development and provision of the cross-section database (http://physics.nist.gov/PhysRefData/Ionization/Xsection.html) operated by the National Institute of Standards and Technology (NIST).

\section{Reference}

Acuña, M. H., and N. Ness, The main magnetic field of Jupiter, J. Geophys. Res., 81 (16), 2917-2922, doi:10.1029/JA081i016p02917, 1976.

Bagenal, F., and J. D. Sullivan, Direct plasma measurements in the lo torus and inner magnetosphere of Jupiter, J. Geophys. Res., 86 (A10), 8447-8466, doi:10.1029/JA086iA10p08447, 1981.

Bagenal, F., Empirical model of the lo plasma torus: Voyager measurements, J. Geophys. Res., 99, 11,043-11,062, doi:10.1029/93JA02908, 1994. 
Bagenal, F., The ionization source near lo from Galileo wake data, Geophys. Res. Lett., 24 (17), 2111-2114, doi:10.1029/97GL02052, 1997a.

Bagenal, F., F. J. Crary, A. I. F. Stewart, N. M. Schneider, D. A. Gurnett, W. S. Kurth, L. A. Frank, and W. R. Paterson, Galileo measurements of plasma density in the lo torus, Geophys. Res. Lett., 24(17), 2119-2122, doi:10.1029/97GL01254, 1997b.

Bagenal, F., and P. A. Delamere, Flow of mass and energy in the magnetospheres of Jupiter and Saturn, J. Geophys. Res., 116, A05209, doi:10.1029/2010JA016294, 2011.

Barabash, S., A. V. Lukyanov, P. C. Brandt, and R. Lundin, Energetic neutral atom imaging of Mercury's magnetosphere 3: Simulated images and insrtument requirements, Planet. Space Sci., 49, 1685-1692, 2001.Barabash, S., et al., The Analyzer of Space Plasmas and Energetic Atoms (ASPERA-3) for the Mars Express mission, Space Sci. Rev., 126 (1), 113-164, 2006.

Barabash, S., et al., The Analyser of Space Plasmas and Energetic Atoms (ASPERA-4) for the Venus Express mission, Planet. Space Sci., 55 (12), 1772-1792, doi:10.1016/j.pss.2007.01.014, 2007.

Barabash, S., et al., Investigation of the solar wind-Moon interaction on board Chandrayaan-1 mission with the SARA experiment, Current Science, 96 (4), 526-532, 2009.

Brandt, P. C., D. G. Mitchell, E. C. Roelof, S. M. Krimigis, C. P. Paranicas, B. H. Mauk, J. Saur, and R. DeMajistre, ENA imaging: seeing the invisible, Johns Hopkins APL technical digest, 26 (2), 143, 2005.

Brown, R. A., Optical line emission from lo, in Exploration of the Planetary System, edited by R. A. Brown, pp. 527-531, Reidel, 1974.

Brown, M. E., Periodicities in the lo plasma torus, J. Geophys. Res., 100, 21,683-21,695, 1995.

Burch, J. L., IMAGE mission overview, Space Sci. Rev., 91 (1-2), 1-14, doi:10.1023/A:1005245323115, 2000.

Burch, J., The first two years of Image, Space Sci. Rev., 109, 1-24, doi:10.1023/B:SPAC.0000007510.32068.68, 2003. 
811 Cheng, A. F., Energetic neutral particles from Jupiter and Saturn, J. Geophys. Res., 91 (A4), 4524-4530, doi:10.1029/JA091iA04p04524, 1986.

813 Connerney, J. E. P., M. H. Acuña, N. F. Ness, and T. Satoh, New models of Jupiter's magnetic field constrained by the lo flux tube footprint, J. Geophys. Res., 103 (A6), 11,929-11,939, doi:10.1029/97JA03726, 1998.

817 Delamere, P. A., A. Steffl, and F. Bagenal, Modeling temporal variability of plasma conditions in the lo torus during the Cassini era, J. Geophys. Res., 109, A10216, doi: 10.1029/2003JA010354, 2004.

DeMajistre, R., E. C. Roelof, P. C. Brandt, and D. G. Mitchell, Retrieval of global magnetospheric ion distributions from high-energy neutral atom measurements made by the IMAGE/HENA instrument, J. Geophys. Res., 109 (A04214), doi:10.1029/2003JA010322, 2004.

Divine, N., and H. B. Garrett, Charged particle distributions in jupiter's magnetosphere, J. Geophys. Res., 88 (A9), 6889-6903, 1983.

Dessler, A. J. (Ed.), Physics of the Jovian Magnetosphere, Cambridge University Press, 1983.

Dessler, A. J., Differential rotation of the magnetic fields of gaseous planets, Geophys. Res. Lett., 12 (5), 299-302, doi:10.1029/GL012i005p00299, 1985.

Frank, L. A., and W. R. Paterson, Survey of thermal ions in the lo plasma torus with the Galileo spacecraft, J. Geophys. Res., 106 (A4), 6131-6149, doi:10.1029/2000JA000159, 2001. Gröller, and A. Mura, Exospheres and energetic neutral atoms of Mars, 
Venus and Titan, Space Sci. Rev., 162, 213-266, doi:10.1007/s11214011-9834-4, 2011.

Futaana, Y., et al., Empirical energy spectra of neutralized solar wind protons from the lunar regolith, J. Geophys. Res., 117 (E5), E05005, doi:10.1029/2011JE004019, 2012.

Futaana, Y., S. Barabash, M. Wieser, C. Lue, P. Wurz, A. Vorburger, A. Bhardwaj, and K. Asamura, Remote energetic neutral atom imaging of electric potential over a lunar magnetic anomaly, Geophys. Res. Lett., 40, 262-266, doi:10.1002/grl.50135, 2013.

Galli, A., et al., Tailward flow of energetic neutral atoms observed at Venus, J. Geophys. Res., 113, E00B15, doi:10.1029/2008JE003096, 2008a.

Galli, A., et al., Tailward flow of energetic neutral atoms observed at Mars, J. Geophys. Res., 113 (E12), E12012, doi:10.1029/2008JE003139, 2008b.

Gladstone, G. R., and D. T. Hall, Recent results from EUVE observations of the lo plasma torus and Jupiter, J. Geophys. Res., 103 (E9), 19,927-19,933, doi:10.1029/98JE00823, 1998.

Goldstein, J., and D. J. McComas, Five years of stereo magnetospheric imaging by TWINS, Space Sci. Rev., doi:10.1007/s11214-013-0012-8, 2013.

Grande, M., Investigation of magnetospheric interactions with the hermean surface, Adv. Space Res., 19(10), 1609-1614, 1997.

Grasset, O., et al., JUpiter ICy moons Explorer (JUICE): An ESA mission to orbit Ganymede and to characterise the Jupiter system, Planet. Space Sci., 78 (0), 1-21, doi:10.1016/j.pss.2012.12.002, 2013.

Gruntman, M., Energetic neutral atom imaging of space plasmas, Rev. Sci. Instrum., 68, 3617, 1997.

Hansen, C. J., D. E. Shemansky, and A. R. Hendrix, Cassini UVIS observations of europa's oxygen atmosphere and torus, Icarus, 176 (2), 305-315, doi:http://dx.doi.org/10.1016/j.icarus.2005.02.007, 2005.Herbert, F., G. R. Gladstone, and G. E. Ballester, Extreme ultraviolet explorer spectra of the lo plasma torus: Improved spectral resolution and new results, J. Geophys. Res., 106(A11), 26,293-26,309, doi:10.1029/2000JA002501, 2001. 
Herbert, F., N. M. Schneider, A. R. Hendrix, and F. Bagenal, Hubble space telescope observations of sulfur ions in the io plasma torus: New constraints on the plasma distribution, J. Geophys. Res., 108(A5), 1167, doi:10.1029/2002JA009510, 2003.

Hill, T. W., A. J. Dessler, and F. C. Michel, Configuration of the jovian magnetosphere, Geophys. Res. Lett., 1(1), 3-6, doi:10.1029/GL001i001p00003, 1974.

Hill, T. W., and F. C. Michel, Heavy ions from the Galilean satellites and the centrifugal distortion of the Jovian magnetosphere, J. Geophys. Res., 81, 4561, doi:10.1029/JA081i025p04561, 1976.

Hill, T. W., Corotation lag in Jupiter's magnetosphere: Comparison of observation and theory, Science, 207 (4428), 301-302, doi:10.1126/science.207.4428.301, 1980.

Itikawa, Y., and A. Ichimura, Cross sections for collisions of electrons and photons with atomic oxygen, J. Phys. Chem. Ref. Data, 19 (3), 637-651, doi:10.1063/1.555857, 1990.

Johnson, R. E., and D. F. Strobel, Charge exchange in the lo torus and exosphere, J. Geophys. Res., 87 (A12), 10,385-10,393, doi:10.1029/JA087iA12p10385, 1982.

Kazama, Y., S. Barabash, A. Bhardwaj, K. Asamura, Y. Futaana, M. Holmström, R. Lundin, R. Sridharan, and P. Wurz, Energetic neutral atom imaging mass spectroscopy of the moon and mercury environments, Advances in Space Research, 37 (1), 38-44, doi:doi: 10.1016/j.asr.2005.05.047, 2006.

Kim, Y. K., and J. P. Desclaux, lonization of carbon, nitrogen, and oxygen by electron impact, Phys. Rev. A, 66, 012708, doi:10.1103/PhysRevA.66.012708, 2002.

Kirsch, E., S. M. Krimigis, J. W. Kohl, and E. P. Keath, Upper limits for X-Ray and energetic neutral particle emission from Jupiter: Voyager-1 results, Geophys. Res. Lett., 8 (2), 169-172, doi:10.1029/GL008i002p00169, 1981. 
Kivelson, M. G., F. Bagenal, W. S. Kurth, F. M. Neubauer, C. Paranicas, and J. Saur, Magnetospheric interactions with satellites, in Jupiter: The Planet, Satellites and Magnetosphere, chap. 21, pp. 513-536, Cambridge University Press, 2004.

Krimigis, S. M., T. P. Armstrong, W. I. Axford, C. O. Bostrom, C. Y. Fan, G. Gloeckler, and L. J. Lanzerotti, The low energy charged particle (LECP) experiment on the Voyager spacecraft, Space Sci. Rev., 21, 329-354, doi:10.1007/BF00211545, 1977.

Krimigis, S. M., et al., A nebula of gases from lo surrounding Jupiter., Nature, 415 (6875), 994-996, doi:10.1038/415994a, 2002.

Kupo, I., Y. Mekler, and A. Eviatar, Detection of ionized sulfur in the Jovian magnetosphere, ApJ, 205, L51-L53, doi:10.1086/182088, 1976.

Lindsay, B. G., and R. F. Stebbings, Charge transfer cross sections for energetic neutral atom data analysis, J. Geophys. Res., 110 (A12), doi:10.1029/2005JA011298, 2005.

Massetti, S., S. Orsini, A. Milillo, A. Mura, E. De Angelis, H. Lammer, and P. Wurz, Mapping of the cusp plasma precipitation on the surface of mercury, Icarus, 166(2), 229-237, 2003.

Mauk, B. H., D. G. Mitchell, S. M. Krimigis, E. C. Roelof, and C. P. Paranicas, Energetic neutral atoms from a trans-Europa gas torus at Jupiter, Nature, 421 (6926), 920-922, doi:10.1038/nature01431, 2003.

McComas, D., et al., IBEX — Interstellar Boundary Explorer, Space Sci. Rev., 146 (1), 11-33, doi:10.1007/s11214-009-9499-4, 2009.

McGrath, M. A., and R. E. Johnson, Charge exchange cross sections for the lo plasma torus, J. Geophys. Res., 94 (A3), 2677-2683, doi:10.1029/JA094iA03p02677, 1989.

McNutt, R. L., J. W. Belcher, J. D. Sullivan, F. Bagenal, and H. S. Bridge, Departure from rigid co-rotation of plasma in jupiter's dayside magnetosphere, Nature, 280 (5725), 803-803, doi:10.1038/280803a0, 1979. 
Mendillo, M., J. Wilson, J. Spencer, and J. Stansberry, Io's volcanic control of Jupiter's extended neutral clouds, Icarus, 170(2), 430-442, doi:10.1016/j.icarus.2004.03.009, 2004.

Mendillo, M., S. Laurent, J. Wilson, J. Baumgardner, J. Konrad, and W. C. Karl, The sources of sodium escaping from lo revealed by spectral high definition imaging, Nature, 448(7151), 330-332, 2007.

Milillo, A., et al., Energetic neutral particles detection in the environment of jupiter's icy moons: Ganymede's and europa's neutral imaging experiment (genie), Planetary and Space Science, 88 (0), 53-63, doi:10.1016/j.pss.2013.08.008, 2013.

Nakano, S., G. Ueno, Y. Ebihara, M.-C. Fok, S. Ohtani, P. C. Brandt, D. G. Mitchell, K. Keika, and T. Higuchi, A method for estimating the ring current structure and the electric potential distribution using energetic neutral atom data assimilation, J. Geophys. Res., 113 (A5), doi:10.1029/2006JA011853, 2008.

Nozawa, H., H. Misawa, S. Takahashi, A. Morioka, S. Okano, and R. Sood, Long-term variability of [SII] emissions from the io plasma torus between 1997 and 2000, J. Geophys. Res., 109(A7), A07,209, doi:10.1029/2003JA010241, 2004.

Orsini, S., A. Milillo, E. D. Angelis, A. M. D. Lellis, V. Zanza, and S. Livi, Remote sensing of mercury's magnetospheric plasma environment via energetic neutral atoms imaging, Planet. Space Sci., 49 (14-15), 16591668, 2001.

Orsini, S., S. Livi, K. Torkar, S. Barabash, A. Milillo, P. Wurz, A. M. Di Lellis, and E. Kallio, Serena: A suite of four instruments (ELENA, STROFIO, PICAM and MIPA) on board BepiColombo-MPO for particle detection in the Hermean environment, Planet. Space Sci., 58 (1-2), 166-181, doi:10.1016/j.pss.2008.09.012, 2010.

Plainaki, C., A. Milillo, A. Mura, S. Orsini, and T. Cassidy, Neutral particle release from europa's surface, Icarus, 210(1), 385-395, 2010.

Rees, M. H., Physics and Chemistry of the Upper Atmosphere, Cambridge University Press, 1989. 
Richards, P. G., J. A. Fennelly, and D. G. Torr, EUVAC: A solar EUV flux model for aeronomic calculations, J. Geophys. Res., 99 (A5), 8981-8992, doi:10.1029/94JA00518, 1994.

Roelof, E., and D. Williams, The terrestrial ring current-from in situ measurements to global images using energetic neutral atoms, Johns Hopkins APL Technical Digest, 9, 144-163, 1988.

Saito, Y., J. A. Sauvaud, M. Hirahara, S. Barabash, D. Delcourt, T. Takashima, and K. Asamura, Scientific objectives and instrumentation of Mercury Plasma Particle Experiment (MPPE) onboard MMO, Planet. Space Sci., 58 (1-2), 182-200, doi:10.1016/j.pss.2008.06.003, 2010.

Sandel, B. R., and A. L. Broadfoot, Discovery of an lo-correlated energy source for lo's hot plasma torus, J. Geophys. Res., 87 (A4), 2231-2240, doi:10.1029/JA087iA04p02231, 1982.

Sandel, B. R., and A. J. Dessler, Dual periodicity of the Jovian magnetosphere, J. Geophys. Res., 93, 5487-5504, 1988.

Schaufelberger, A., et al., Scattering function for energetic neutral hydrogen atoms off the lunar surface, Geophys. Res. Lett., 38, L22202, doi:10.1029/2011GL049362, 2011.

Schmidtke, G., F. G. Eparvier, S. C. Solomon, W. K. Tobiska, and T. N. Woods, The TIGER (thermospheric-ionospheric geospheric research) program: Introduction, Adv. Space Res., 37 (2), 194-198, doi:http://dx.doi.org/10.1016/j.asr.2005.02.088, 2006.

Schneider, N. M., J. T. Trauger, J. K. Wilson, D. I. Brown, R. W. Evans, and D. E. Shemansky, Molecular origin of Io's fast sodium, Science, 253 (5026), 1394-1397, doi:10.1126/science.253.5026.1394, 1991.

Schneider, N. M., and F. Bagenal, lo's neutral clouds, plasma torus, magnetospheric interaction, in Io After Galileo: A new view of Jupiter's Volcanic Moon, edited by R. M. C. Lopes and J. R. Spencer, pp. 265286, Springer, 2007.

Smyth, W. H., and M. R. Combi, A general model for lo's neutral gas clouds. I Mathematical description, Ap/ Suppl. Ser., 66, 397-411, doi:10.1086/191264, 1988. 
Smyth, W. H., Neutral cloud distribution in the Jovian system, Adv. Space Res., 12 (8), 337-346, 1992.

Smyth, W. H., and M. Marconi, Nature of the iogenic plasma source in Jupiter's magnetosphere I. Circumplanetary distribution, Icarus, 166 (1), 85-106, doi:10.1016/S0019-1035(03)00176-3, 2003.

Steffl, A. J., A. I. F. Stewart, and F. Bagenal, Cassini UVIS observations of the lo plasma torus: I. initial results, Icarus, 172(1), 78-90, doi: 10.1016/j.icarus.2003.12.027, 2004a.

Steffl, A. J., F. Bagenal, and A. I. F. Stewart, Cassini UVIS observations of the Io plasma torus: II. radial variations, Icarus, 172(1), 91-103, doi: 10.1016/j.icarus.2004.04.016, 2004b.

Steffl, A. J., P. A. Delamere, and F. Bagenal, Cassini UVIS observations of the Io plasma torus: III. observations of temporal and azimuthal variability, Icarus, 180 (1), 124-140, doi:10.1016/j.icarus.2005.07.013, 2006.

Steffl, A. J., P. A. Delamere, and F. Bagenal, Cassini UVIS observations of the lo plasma torus: IV. modeling temporal and azimuthal variability, Icarus, 194 (1), 153-165, doi: 10.1016/j.icarus.2007.09.019, 2008.

Thomas, N., F. Bagenal, T. W. Hill, and J. K. Wilson, The lo neutral clouds and plasma torus, in Jupiter: The Planet, Satellites and Magnetosphere, chap. 23, pp. 561-591, Cambridge University Press, 2004.

Wang, K., R. M. Thorne, R. B. Horne, and W. S. Kurth, Cold torus whistlers: An indirect probe of the inner jovian plasma- sphere, J. Geophys. Res. 103, 14 987-14 994, 1998a.

vVang, K., R. I'vi. Thorne, R. B. Horne, and W. S. Kurth, Constraints on jovian plasma properties from a dispersion analysis of unducted whistlers in the warm lo torus, J. Geophys. Res. 103, 14 979-14 986, $1998 \mathrm{~b}$.

Wieser, M., et al., Extremely high reflection of solar wind protons as neutral hydrogen atoms from regolith in space, Planet. Space Sci., 57, 2132 2134, doi:10.1016/j.pss.2009.09.012, 2009.

Wilson, J., M. Mendillo, J. Baumgardner, N. Schneider, J. Trauger, and B. Flynn, The dual sources of Io's sodium clouds, Icarus, 157 (2), 476-489, doi:10.1006/icar.2002.6821, 2002. 
Wurz, P., Detection of energetic neutral particles, in The Outer Heliosphere: Beyond the Planets, edited by K. Scherer, H. Fichtner, and E. Marsch, pp. 251-288, Copernicus Gesellschaft e. V., Katlenburg-Lindau, 2000.

Yoneda, M., M. Kagitani, and S. Okano, Short-term variability of jupiter's extended sodium nebula, Icarus, 204(2), 589-596, doi: 10.1016/j.icarus.2009.07.023, 2009.

Yoneda, M., H. Nozawa, H. Misawa, M. Kagitani, and S. Okano, Jupiter's magnetospheric change by Io's volcanoes, Geophys. Res. Lett., 37(11), L11,202, doi:10.1029/2010GL043656, 2010.

Yoneda, M., et al., Mid-infrared observations of Io's volcanism from the ground in 2011 and 2012, Icarus, 236, 153-156, doi: 10.1016/j.icarus.2014.01.019, 2014.

Zarka, P., J. Queninnec, and F. J. Crary, Low-frequency limit of jovian radio emissions and implications on source locations and lo plasma wake, Planet. Space Sci. 49, 1137-1149, 2001.

\section{Appendix A: ENA flux calculation under the influence of a gravity field}

The ENA flux, $j_{\mathrm{ENA}}\left[\mathrm{cm}^{-2} \mathrm{~s}^{-1} \mathrm{sr}^{-1} \mathrm{eV}^{-1}\right]$ is normally calculated as a line-of-sight integral of ENA generation:

$$
j_{\mathrm{ENA}}(E)=\int_{-\infty}^{0} j_{\text {plasma }}(E, \hat{l}) \cdot N_{n}\left(l_{10} \dot{105}^{2} \dot{3} d l\right.
$$

where, $j_{\text {plasma }}$ denotes the differential flux of plasma, $E$ is the energy, $I$ is the distance along the direction of flight of the ENAs, and $\hat{l}$ is the unit vector thereof. $N_{n}$ and $\sigma$ are the density of the neutral atoms and the cross section for the charge-exchange process, respectively. Equivalently, one may use the velocity distribution function, $f_{\mathrm{ENA}}\left[\mathrm{s}^{3} / \mathrm{m}^{6}\right]$, for the following formulation:

$$
f_{\mathrm{ENA}}(\vec{v})=\int_{-\infty}^{0} f_{\text {plasma }}(v, \hat{l}) \cdot N_{n}(l)^{1059} \cdot \frac{\sigma}{1060} d l
$$


1061 where $v$ is the velocity vector of the ENAs and $v$ is their speed. $f_{\text {plasma }}$ is the 1062 velocity distribution function of the plasma. In the following, the Expression (A2) 1063 is preferentially applied because although the velocity distribution functions are 1064 conserved by the translation of reference frames in velocity space, the 1065 differential flux is not.

1066 Expressions of (A1) and (A2) can be used under the following assumptions:

1067 1. the change in energy and direction during the charge exchange is negligibly 1068 small, 2. there is no loss of ENAs during the flight, 3. the observer is stationary, 1069 and 4. the trajectories of the ENAs are straight. The first assumption is, in most 1070 of the higher energy range, satisfied for the LENA case. For low energy images 1071 (say <30 eV), one has to in theory modify the energy loss and directional 1072 change to calculate the ENA flux. Under this circumstance, due to the angular 1073 spread, the image will be more "blurred". It is also noted that this effect should 1074 be visible in the frame moving together with the target neutrals. In the lo tori 1075 imaging case, the observer (spacecraft) is moving with respective to the lo 1076 neutral torus, the blurring effect will be less significant. In addition, the main 1077 peak of the lo tori ENAs is $>100 \mathrm{eV}$ for $\mathrm{S}$ and $\mathrm{O}$, we neglect the energy loss and 1078 the directional change. The second assumption is not trivially negligible, but it 1079 is insignificant for lo-tori imaging (see section 3.2). To satisfy the third 1080 assumption, one should properly compensate for the spacecraft velocity (to be 1081 discussed below). The final assumption may be violated in the case of the lo 1082 torus imaging. The trajectories of low-speed LENAs are bent by the gravity of 1083 Jupiter, so (A2) should be modified.

1084 Because no centrifugal nor Corioli's forces contribute explicitly, an inertial 1085 frame is used for the formulation. Under a gravitational field, the ENA 1086 production should be assessed along a ballistic trajectory. For the spacecraft 1087 position $\vec{r}_{0}$ and ENA velocity $\vec{v}_{0}$ to be observed, the ENA trajectory, $L=L\left(\vec{r}_{0}, \vec{v}_{0}\right)$, 1088 can be derived uniquely from the integral (backward in time) of the Newtonian 1089 equation of motion.

1090 Let us now consider a small path length, $\mathrm{d} /$, at a distance of $/$ along the 1091 trajectory $L$. The position $\left(\vec{r}_{1}\right)$ and velocity $\left(\vec{v}_{1}\right)$ at distance $/$ are determined by 1092 integrating the Newtonian equation of motion. Both are functions of $I$, namely, 
$1093 \vec{r}_{1}=\vec{r}_{1}(l)$ and $\vec{v}_{1}=\vec{v}_{1}(l)$. The time at $/$ is also determined from the Newtonian 1094 equation of motion: $t_{1}=t_{1}(l)$. Because the flying time of the ENA from the 1095 generation point to the spacecraft is typically several hours, which cannot be 1096 disregarded considering the time scale of the change in the environment 1097 (corotation is $\sim 10$ hours), the simulation must consider that ENA arriving at time $1098 \mathrm{t}_{0}$ are generated at different timing $\left(\mathrm{t}_{1}\right)$ along the ballistic trajectory depending on 1099 I. The ENA flux production, $\mathrm{d} f_{\mathrm{ENA}}$, from this path length, $\mathrm{d} /$, is then

$$
d f_{\mathrm{ENA}}\left(\overrightarrow{r_{1}}, \overrightarrow{v_{1}} ; t 1\right)=f_{\text {plasma }}\left(\overrightarrow{r_{1}}, \overrightarrow{v_{1}} ; t 1\right) \cdot \sigma \cdot N_{n}\left(\overrightarrow{r_{1}} \dot{1} \delta 0\right) d l
$$

All quantities should be calculated at time of $t_{1}$. We assume no energy loss during the charge exchange, namely, the source plasma velocity is also $\vec{v}_{1}$.

According to the Liouville theorem, the distribution function along the 1104 orbit is conserved. Thus, the integration of (A3) over the distance / yields the 1105 total ENA flux along the trajectory $L$.

$$
f_{\mathrm{ENA}}\left(\vec{r}_{0}, \vec{v}_{0} ; t_{0}=0\right)=\int \mathrm{d} f_{\mathrm{ENA}}\left(\vec{r}_{1}, \vec{v}_{1} ; t_{1}\right)=\int_{-\infty}^{0} f_{\text {plasma }}\left(\vec{r}_{1}, \vec{v}_{1} ; t_{1}\right) \cdot \sigma \cdot N_{n}\left(\vec{r}_{1} ; t_{1}\right) \mathrm{d} l
$$

1107 Expression (A4) describes the ENA velocity distribution function. A 1108 spacecraft is typically moving in an inertial frame, so the spacecraft velocity 1109 should be considered. We define the spacecraft (SC) coordinate system that 1110 moves with the spacecraft velocity, $\vec{V}_{\text {sc }}$. The velocities in the inertial $(\vec{v})$ and SC $1111\left(\vec{v}^{\prime}\right)$ frames are related as follows: $\vec{v}^{\prime}=\vec{v}+\vec{V}_{\mathrm{sc}}$. The velocity distribution function 1112 does not depend on the frame of the reference: $f(\vec{r}, \vec{v})=f^{\prime}\left(\vec{r}^{\prime}, \vec{v}^{\prime}\right)$. Thus, the ENA 1113 velocity distribution function observed at the spacecraft can be described as

$$
f_{\mathrm{ENA}}^{\prime}\left(\vec{r}_{0}^{\prime}=0, \vec{v}_{0}^{\prime} ; t=0\right)=f_{\mathrm{ENA}}\left(\vec{r}_{0}, \vec{v}_{0} ; t=0\right)
$$

The differential flux to be observed can be derived from the velocity distribution function.

$$
j_{\mathrm{ENA}}^{\prime}\left(\vec{r}_{0}^{\prime}=0, E_{0}^{\prime}, \Omega_{0}^{\prime}\right)=\frac{2 E_{0}^{\prime}}{m^{2}} f_{\mathrm{ENA}}^{\prime}\left(\vec{r}_{0}^{\prime}=0, \vec{v}_{0}^{\prime}\right)=\frac{2 E_{0}^{\prime}}{m^{2}} f_{\mathrm{ENA}}\left(\vec{r}_{0}, \vec{v}_{0}\right)
$$

Here, energy and direction $\left(E_{0}{ }^{\prime}, \Omega^{\prime}{ }_{0}\right.$ ) can be calculated from $\vec{v}_{0}^{\prime}$ (and vice versa), and $m$ is the mass of ENAs. In the rightmost expression, the energy is given in the SC frame $\left(E_{0}{ }^{\prime}\right)$. 


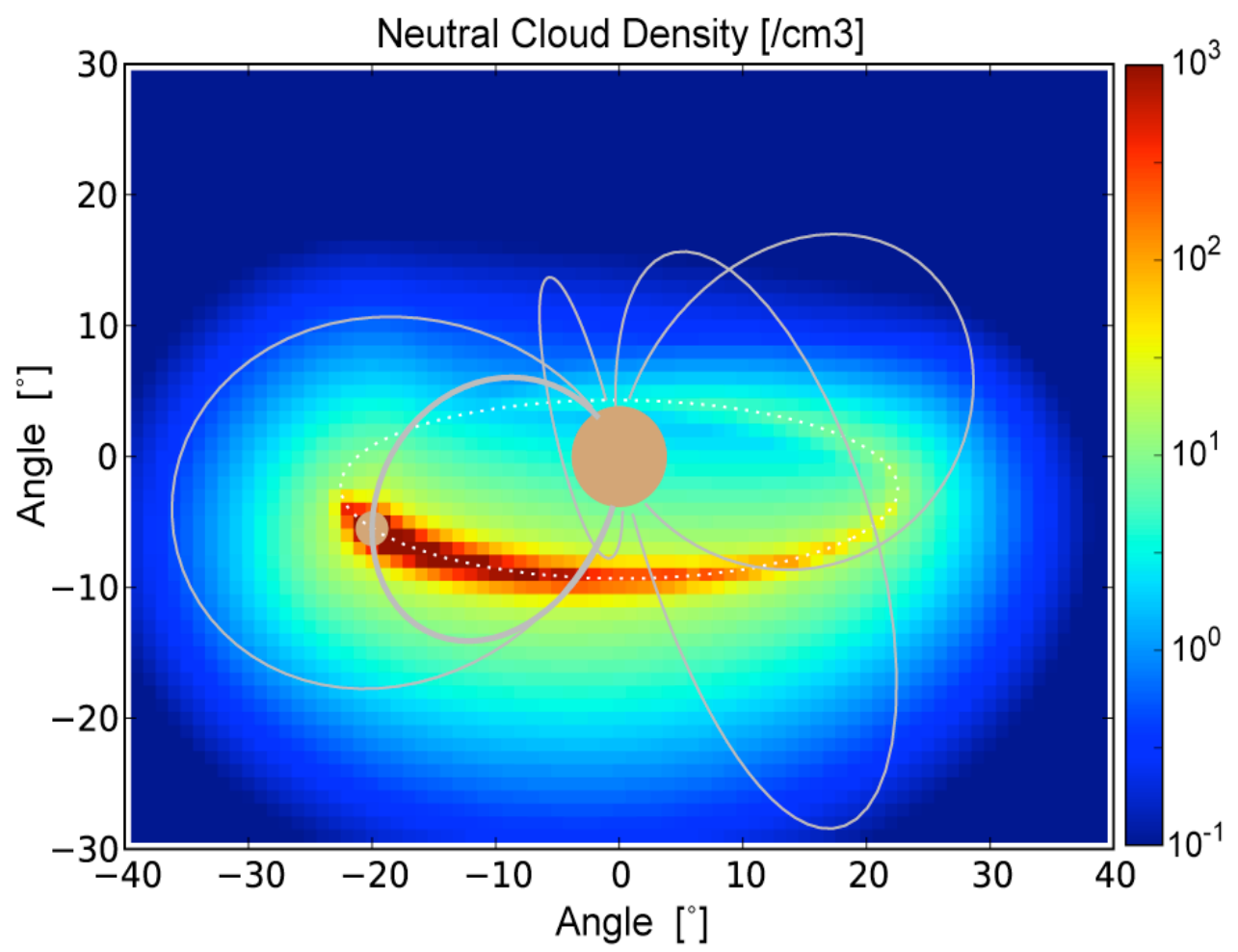

1122

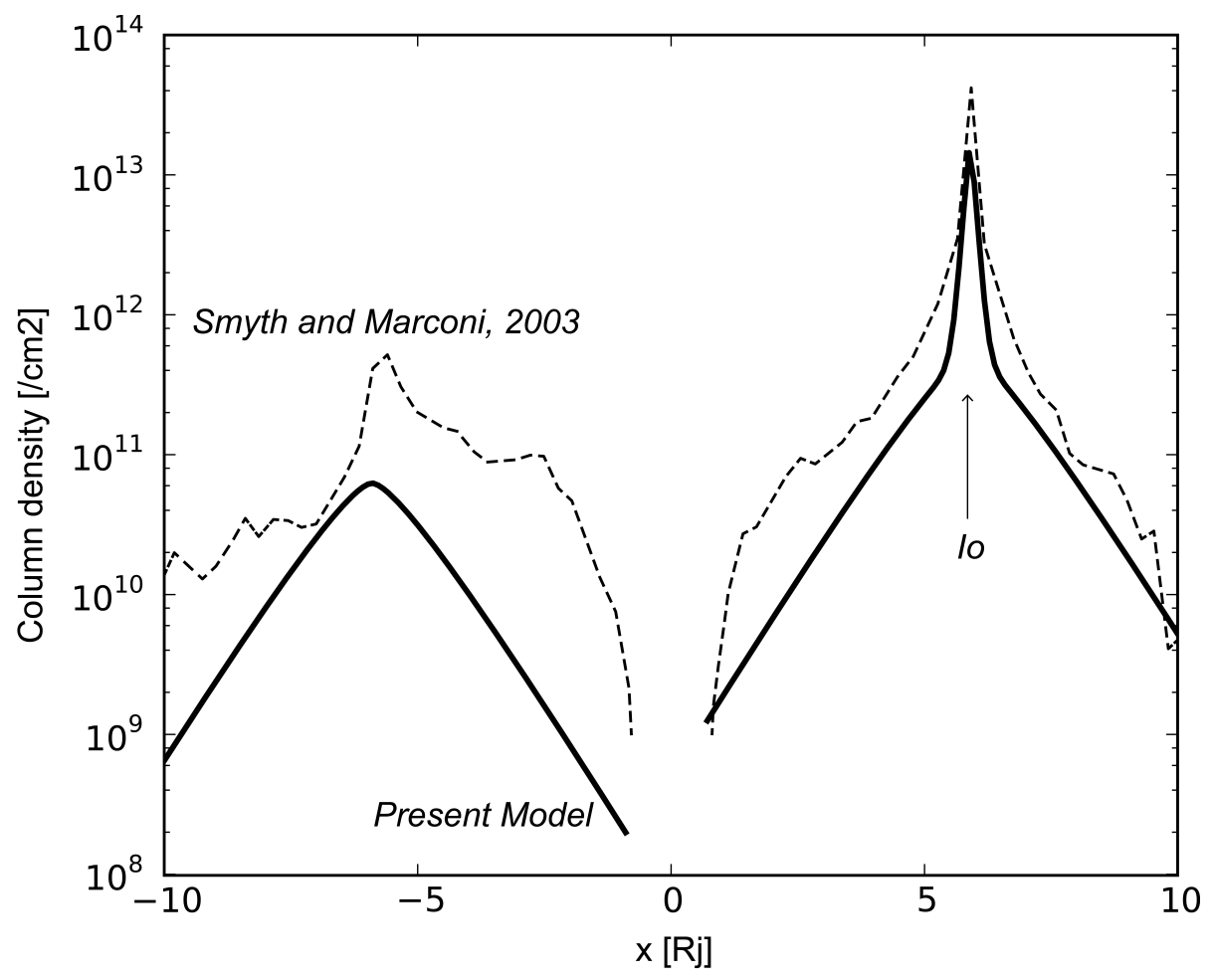

1125 Figure 1: a) Image of the density model of the lo neutral torus used in this paper.

1126 A virtual spacecraft was placed at $\sim 15$ Rj from Jupiter. The spacecraft is above 1127 the equatorial plane at $\sim 16^{\circ}$ north. The dipole magnetic field lines $(L=10)$ are 1128 shown as thin gray lines. The field line that is connected to lo (small circle; not 1129 to scale) is depicted with a thick line. Jupiter, located at the center, is drawn to 
1130 scale. b) Comparison of our model (thick line) and the result obtained by Smyth 1131 and Marconi (2003; dashed line). The column density integrated along the $z$ 1132 axis (perpendicular to the lo orbital plane) is shown as a function of the Jupiter1133 lo distance along the Jupiter-lo line.

1134 


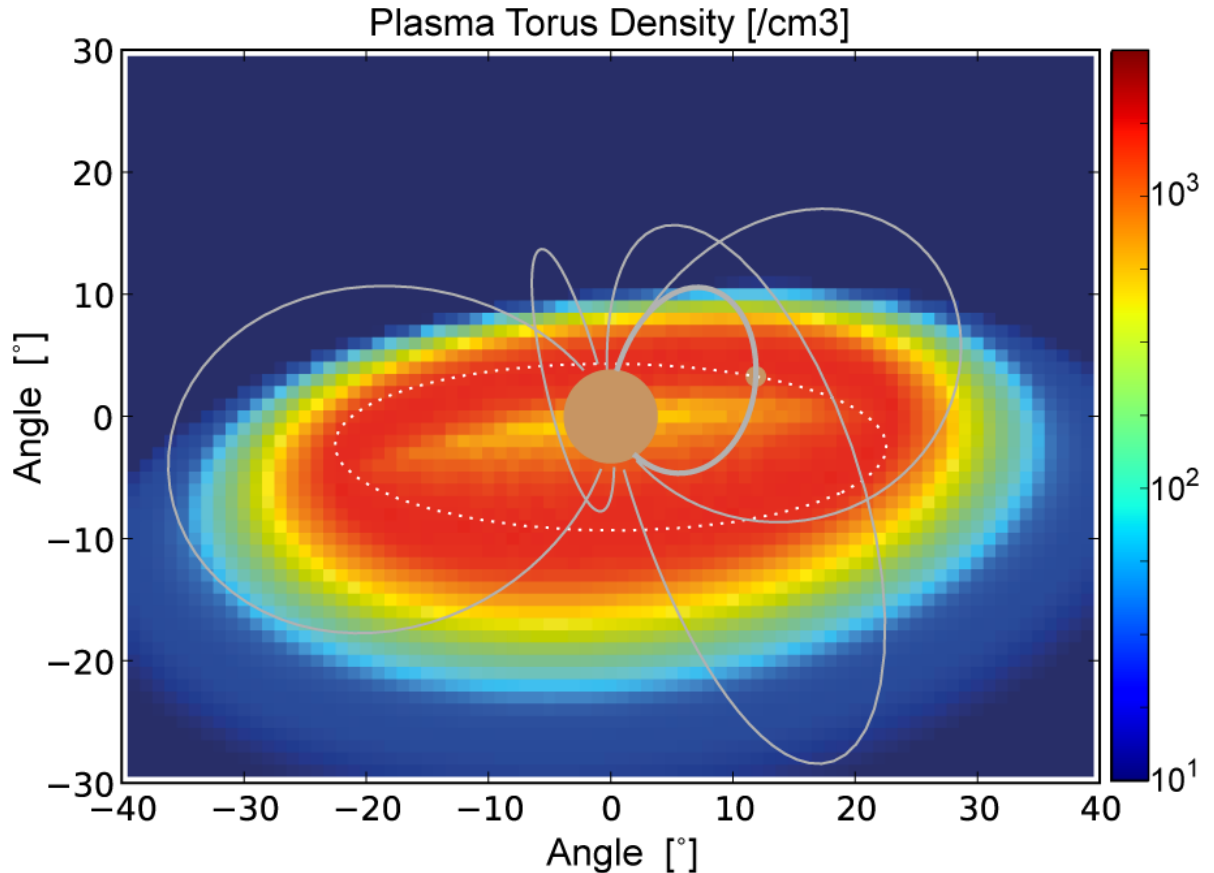

\section{Equatorial density}

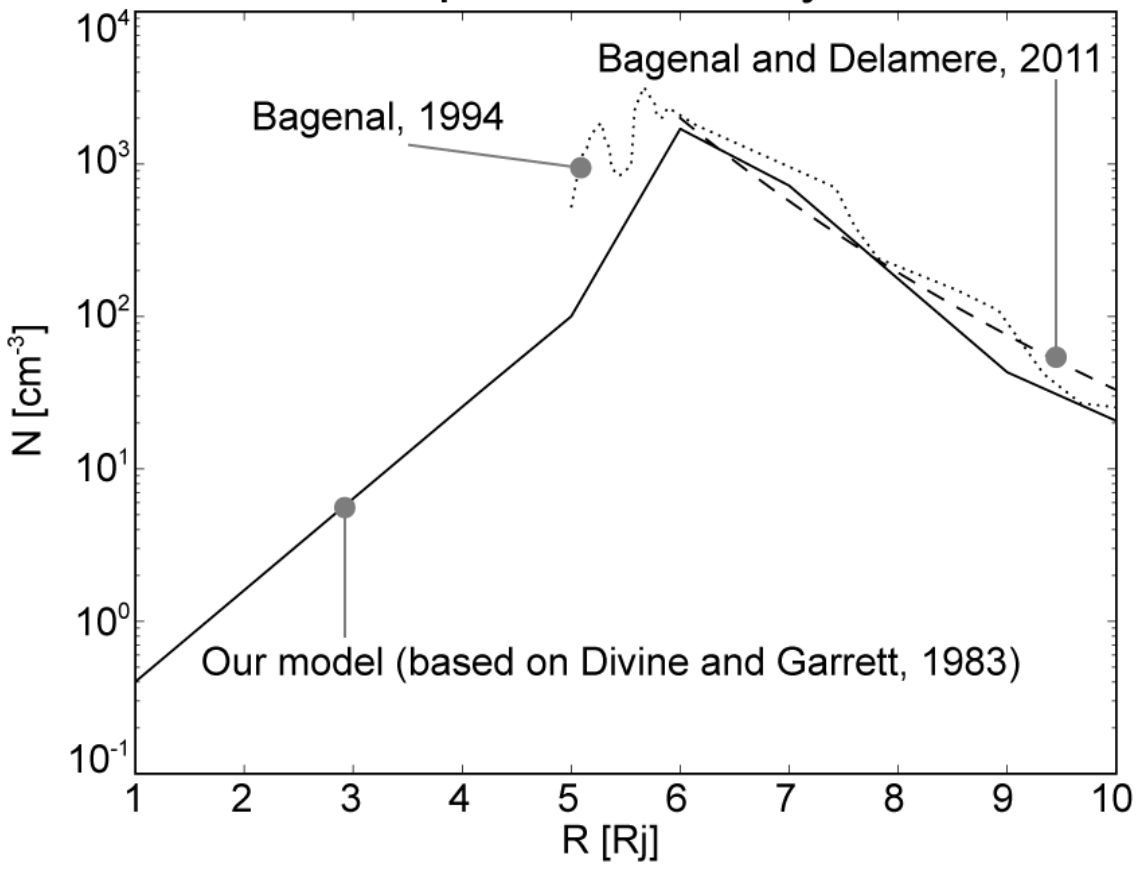

1138 Figure 2: a) Plasma density employed in the plasma-torus model in the same

1139 format as Figure 1a. b) The plasma density at the centrifugal equator as a 1140 function of the distance from the Jovian center. For comparison, two models 1141 (Bagenal,1994 and Bagenal and Delamere, 2011) are shown. 

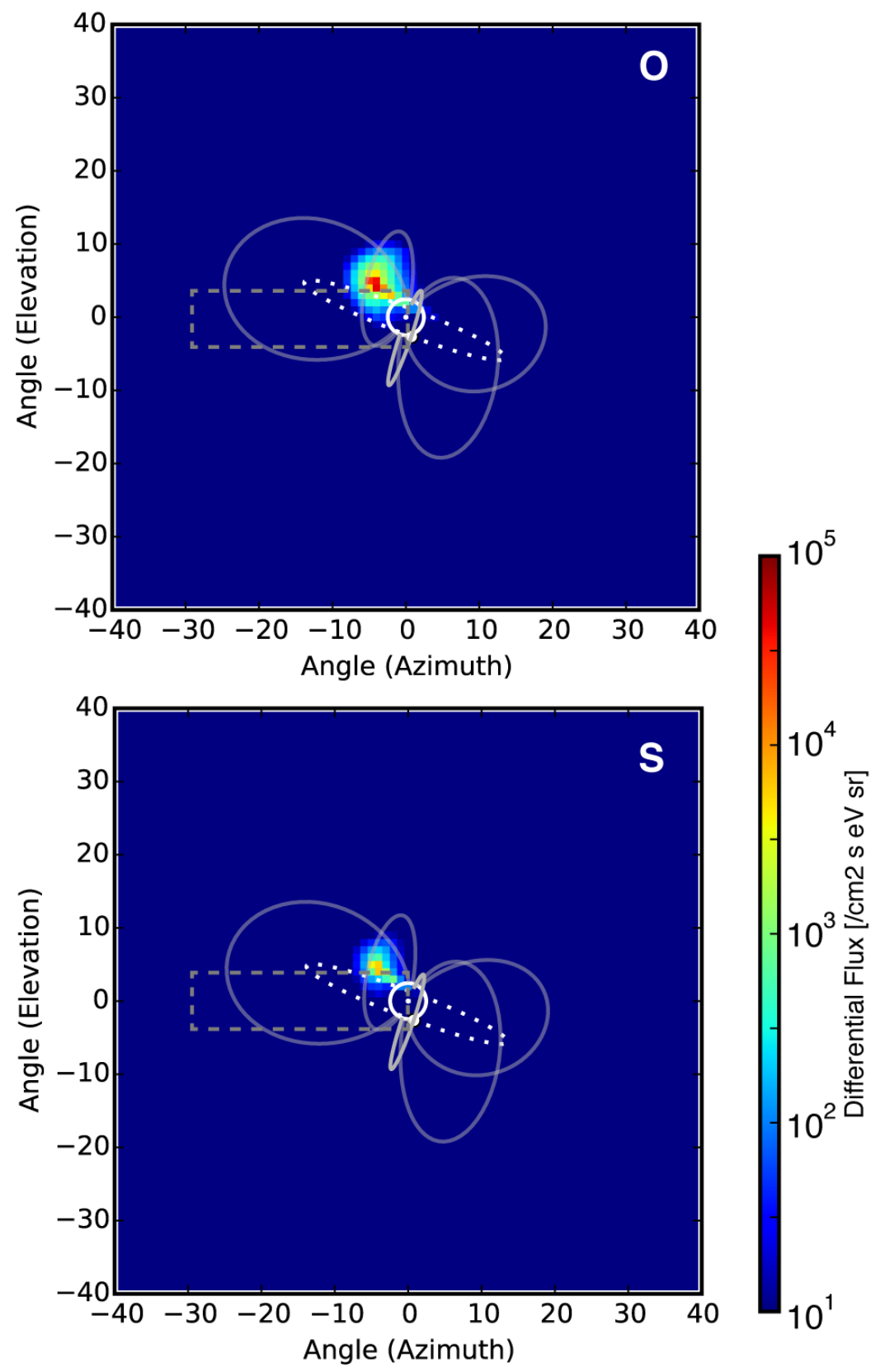

1145 Figure 3: The expected oxygen and sulfur LENA images for an energy of $210 \mathrm{eV}$

1146 and $420 \mathrm{eV}$. The spacecraft was located at $23.3 \mathrm{Rj}$ at a latitude of $6.5^{\circ}$ north 1147 from the Jovian equatorial plane as an example. The spacecraft-lo-Jupiter line is 1148 nearly co-aligned in the projection plane (Jupiter equatorial plane). The open 1149 circle at the origin is Jupiter (to scale). The thin gray curves are Jupiter's 1150 magnetic field lines for $L=10$. The location of lo at the time of observation is 1151 indicated by the filled circle in front of Jupiter, but it is not to scale; the real size 1152 of lo is considerably smaller. The magnetic field line of Jupiter that crosses the 1153 body of lo is represented by the white curve. The dotted white circle 
1154 corresponds to the lo orbit. The sensor's field of view (see Table 2), which is 1155 used for Figure 5, is indicated by the gray dotted rectangle.

1156 

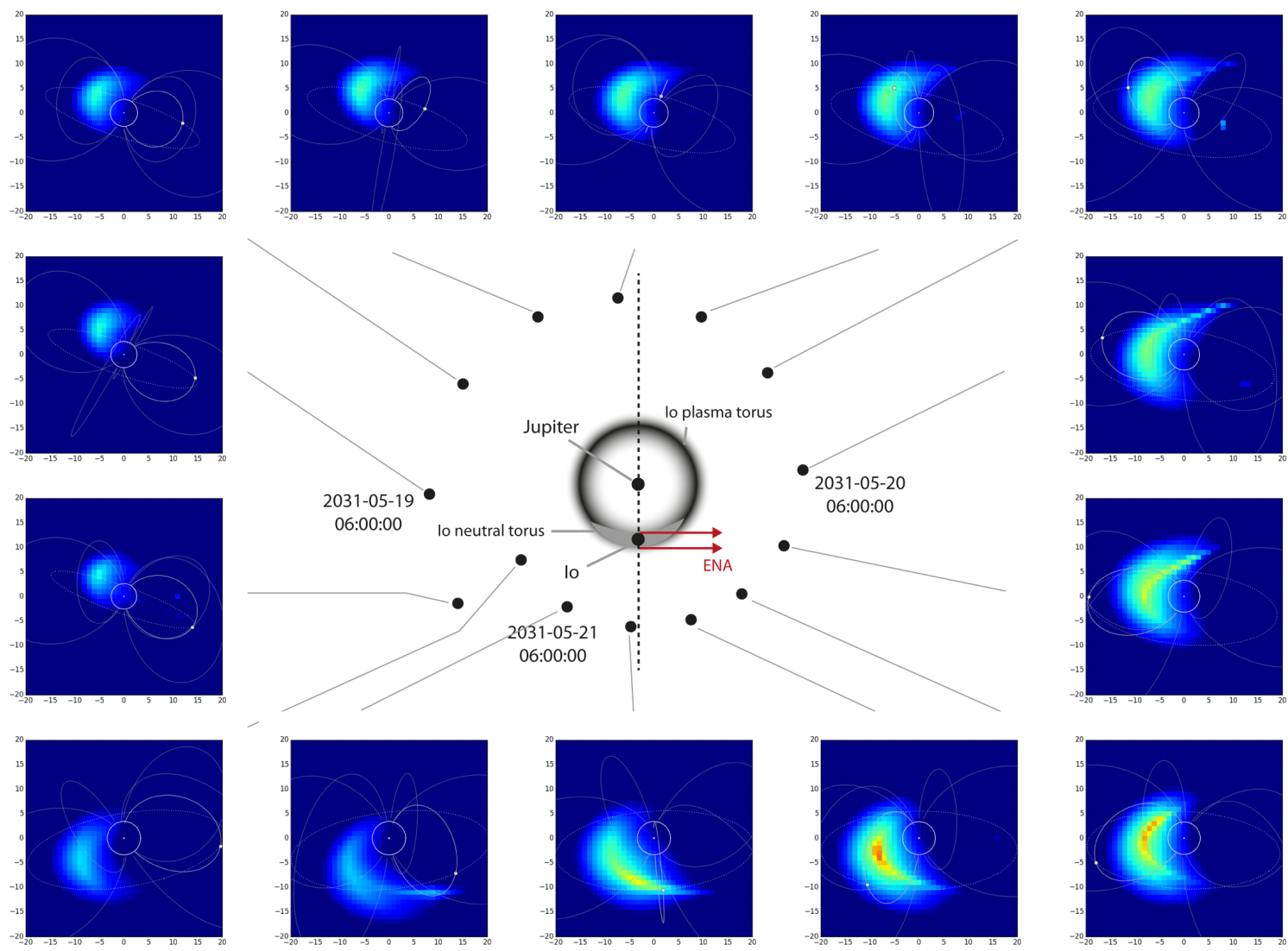

Figure 4: Time series of simulated oxygen LENA images with energy of $210 \mathrm{eV}$.

1159 Illustration at the center is the geometry of the calculation. The Jupiter-centered

1160 lo fixed frame is used. In this frame, the LENA is emitted always in the same

1161 direction (red arrows). Spacecraft location every 4 hours are depicted by filled

1162 dots. Each panel shows the LENA image in the same format as Figure 3, but 1163 zoomed into 20x20 degrees.

1164 


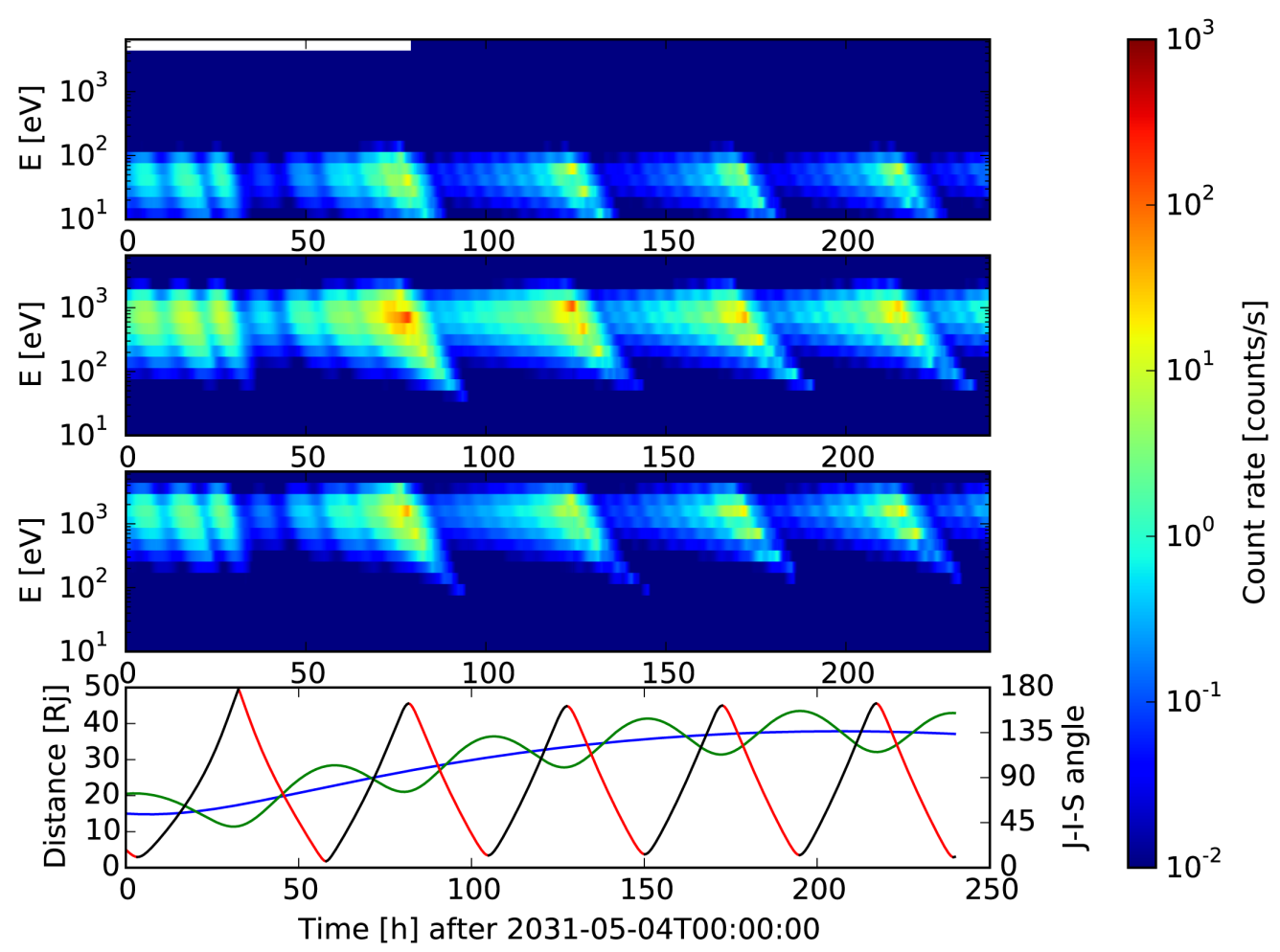

1165

1166 Figure 5: a-c) Calculated energy-time spectrograms for three species of LENAs

$1167(H, O$, and S). The preliminary JUICE orbit and the geometric factor of the JNA-

1168 prototype are used to calculate the count rates. A sensor pixel of $30 \times 5^{\circ}$ is used

1169 (see white box in Figure 3). d) The geometry of the spacecraft, Io, and Jupiter.

1170 The blue and green solid lines are the distances of the spacecraft from Jupiter

1171 and Io, respectively. The black and red solid lines represent the Jupiter-lo-

1172 spacecraft angle. A black (red) line indicates that lo is located on the toward

1173 (away) side, as seen from the spacecraft. 


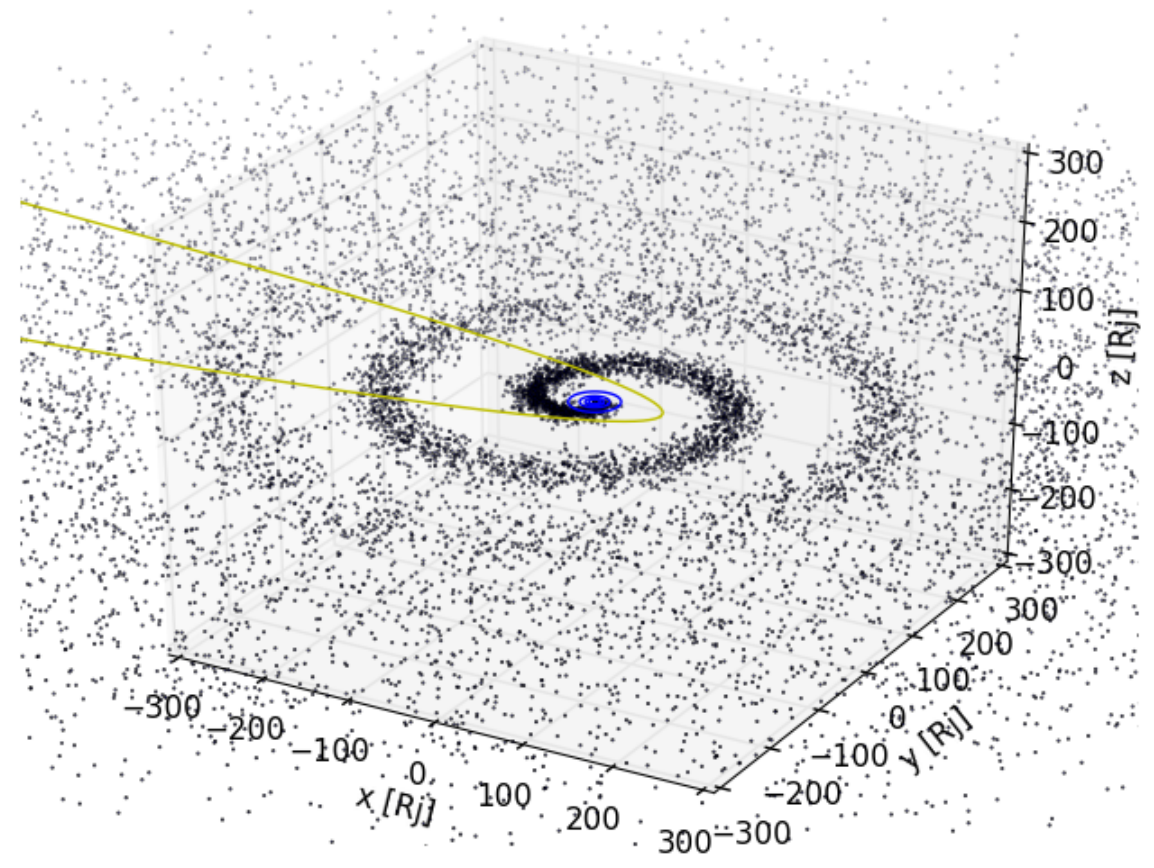

1176 Figure 6: A snapshot of a 3-D view of the spatial distribution of LENAs from the 1177 lo torus. Each dot represents a charge-exchanged LENA. The four blue circles in 1178 the center correspond to the orbits of the Galilean moons (the innermost circle 1179 corresponds to lo). A very simplified shape of the Jovian magnetopause (in the 1180 equatorial plane of Jupiter) is shown for reference. 
1182 Table 1: Charge-exchange cross sections in the units of $10^{-16} \mathrm{~cm}^{2}$ for each plasma 1183 species at a relative velocity of $\sim 60 \mathrm{~km} / \mathrm{s}$. For hydrogen- and proton-related 1184 reactions, we refer to Lindsay and Stebbings (2005). For other reactions, data 1185 from McGrath and Johnson (1989) are used. The convolved cross section for 1186 each ion species are first calculated by taking the weighted average of the 1187 neutral species. Then, taking the weighted average the relative fraction of the 1188 parent ion species in different charge state, the total cross section for each LENA 1189 species are calculated. Reactions for which cross sections are unavailable are 1190 disregarded for the calculation of total cross sections.

\begin{tabular}{|c|c|c|c|c|c|c|c|c|c|}
\hline & $\mathbf{H}$ & 0 & $S$ & 02 & so & SO2 & $\begin{array}{l}\text { Convolved } \\
\text { cross } \\
\text { section }\end{array}$ & Fraction & $\begin{array}{l}\text { Total } \\
\text { cross } \\
\text { section }\end{array}$ \\
\hline H+ & 39 & 11 & & 10 & & & 11.8 & 0.1 & 11.8 \\
\hline O+ & 11 & 22 & 11.2 & 13 & 1.6 & 11 & 18.9 & 0.4 & \\
\hline O++ & & $\begin{array}{c}9.8 \\
6\end{array}$ & 39 & & & & 14.1 & 0.01 & 18.8 \\
\hline S+ & & 0 & 29 & 0 & 50 & 0 & 5.2 & 0.1 & \\
\hline S++ & & $\begin{array}{l}12 . \\
15\end{array}$ & 13.5 & & & & 11.2 & 0.15 & 8.8 \\
\hline $\begin{array}{c}\text { Fracti } \\
\text { on }\end{array}$ & $\begin{array}{c}0.5 \\
6\end{array}$ & 4 & 1 & 0 & 0 & 0 & & & \\
\hline
\end{tabular}


1193 Table 2: Specifications of the LENA sensor (JNA-prototype) used to calculate the 1194 count rate from the physical quantities. The parameters are based on the 1195 predecessor sensors of the JNA-prototype, namely, ENA, mounted on 1196 BepiColombo/MMO and CENA mounted on Chandrayaan-1 (Kazama et al., 1197 2006; Barabash et al., 2009).

1198

\begin{tabular}{|c|c|}
\hline Angular resolution & $30^{\circ} \times 5^{\circ}$ \\
\hline Aperture & $150^{\circ} \times 7^{\circ}$ \\
\hline Energy range & $10-3000 \mathrm{eV}$ \\
\hline Mass resolution & $\mathrm{H}, \mathrm{He}, \mathrm{O}, \mathrm{S},>\mathrm{S}$ \\
\hline Geometric factor (incl. efficiency) & $5 \times 10 \mathrm{~cm} \mathrm{sr} \mathrm{eV} / \mathrm{eV}$ \\
\hline Energy resolution (DE/E) & $100 \%$ \\
\hline
\end{tabular}

1199

1200 
1201 Table 3: Characteristic quantities that can be retrieved from Io-torus LENA 1202 imaging.

1203

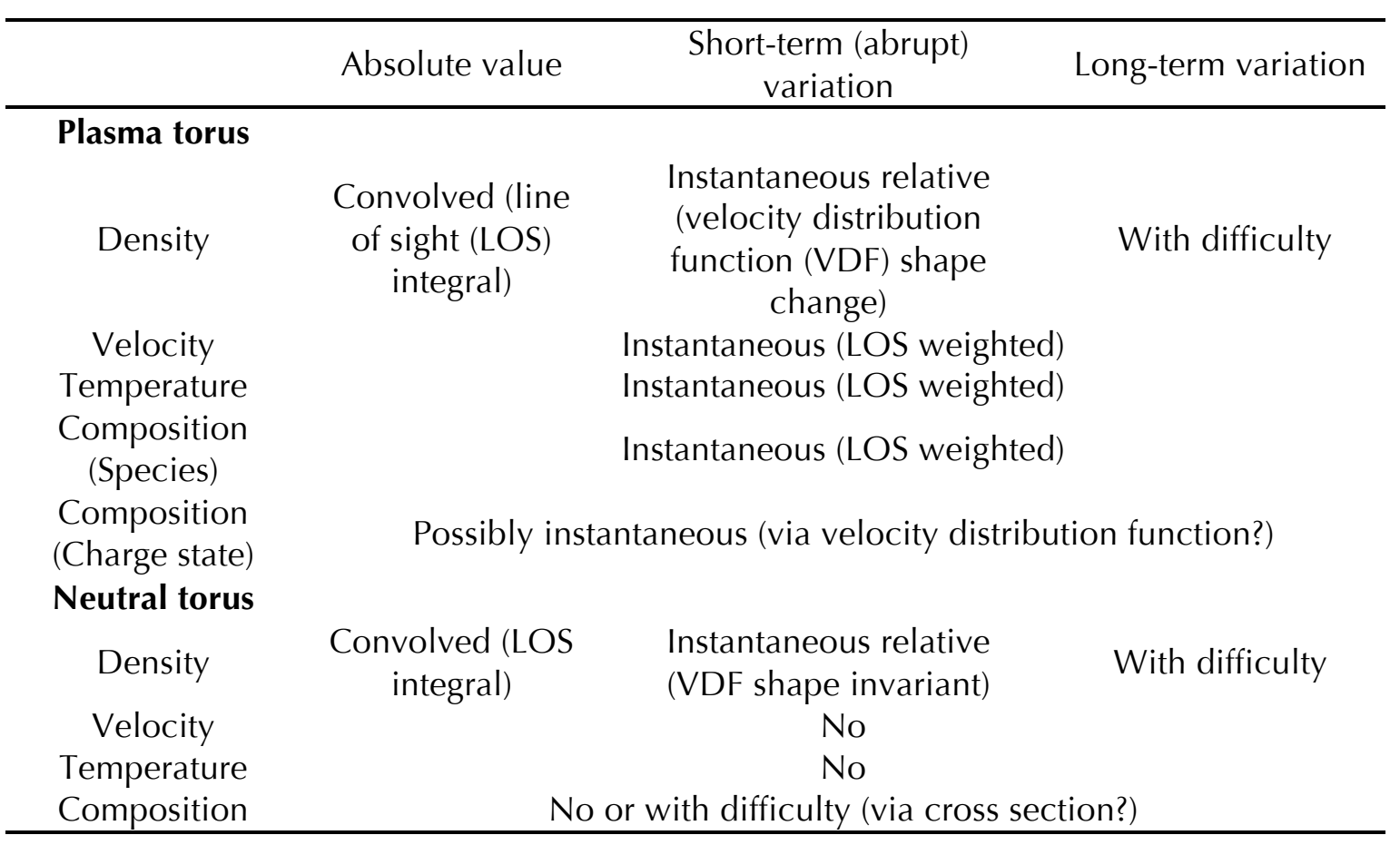


1205 